KODAI MATH. SEM. REP.

20 (1968), 318-354

\title{
DIFFERENTIAL GEOMETRY OF TANGENT BUNDLES OF ORDER 2
}

\author{
By Kentaro Yano and Shigeru Ishihara \\ Dedicated to Professor Shisanji Hokari on his Sixtieth Birthday'
}

\section{§ 0. Introduction.}

The differential geometry of tangent bundles has been studied by Davies [13], Dombrowski [1], Kobayashi [15], Ledger [2], [3], [16], Morimoto [4], [5], Okumura [8], Sasaki [6], Tachibana [8], Tanno [9], Tondeur [10], the present authors [2], [3], [11], [13], [14], [15], [16], [17], [18] and others and that of cotangent bundles by Patterson [17], [18], Satô [7] and one of the present authors [12], [17], [18].')

The purpose of the present paper is to study the differential geometry of tangent bundles of order 2, the tangent bundle of order $2 T_{2}(M)$ of a differentiable manifold $M$ being defined as the set of all 2 -jets of $M$ determined by mappings of the real line $R$ into $M$.

In $\S 1$, we define the tangent bundles of order 2 and induced coordinates in it and fix the notations used throughout the paper.

In $\S 2$, we study the lifts of functions and two vector fields $A$ and $B$ existing a priori in $T_{2}(M)$.

$\S 3$ is devoted to the study of lifts of vector fields, 1 -forms and derivations, and $\S 4$ to the study of lifts of tensor fields and two linear mappings $\alpha$ and $\beta$. In $\S 5$, we give the local expressions of these lifts.

In $\S 6$, we study in more detail the lifts of tensor fields of type $(1,1)$ and discuss lifts of torsion tensors and Nijenhuis tensors.

$\S 7$ is devoted to the study of lifts of affine connections and also of curvature tensor and torsion tensor of the connection.

We study lifts of infinitesimal transformations in $\S 8$ and geodesics in $T_{2}(M)$ in the last $\S 9$.

\section{Contents}

$\S 1$. Tangent bundles of order 2 .

$\S 2$. Lifts of functions.

$\S 3$. Lifts of vector fields, 1 -forms and derivations.

$\S 4$. Lifts of tensor fields.

$\S 5$. Local expressions.

Received November 27, 1967.

1) The numbers in brackets [ ] refer to Bibliography at the end of the paper. 
$\S 6$. Lifts of tensor fields of type $(1,1)$.

$\S 7$. Lifts of affine connections.

$\S 8$. Lifts of infinitesımal transformations.

§9. Geodesics.

\section{$\S 1$. Tangent bundles of order 2 .}

Let $M$ be a differentiable manifold ${ }^{2)}$ of dimension $n$ and $R$ the real line. We introduce an equivalence relation $\sim$ in the set of all differentiable mappings $F: R \rightarrow M$ as follows. Let $r \geqq 1$ be a fixed integer. If two differentiable mappings $F: R \rightarrow M$ and $G: R \rightarrow M$ satisfy the conditions ${ }^{3)}$

$$
F^{h}(0)=G^{h}(0), \quad \frac{d F^{h}(0)}{d t}=\frac{d G^{h}(0)}{d t}, \quad \cdots, \quad \frac{d^{r} F^{h}(0)}{d t^{r}}=\frac{d^{r} G^{h}(0)}{d t^{r}},
$$

the mappings $F$ and $G$ being respectively represented by $x^{h}=F^{h}(t)$ and $x^{h}=G^{h}(t)$ $(t \in R)$ with respect to local coordinates $\left(x^{h}\right)$ defined in a coordinate neighborhood containing the point $F^{h}(0)=G^{h}(0)$, then we say that the two mappings $F$ and $G$ are equivalent to each other and write $F \sim G$. Each equivalence class determined by the equivalence relation $\sim$ is called briefly an $r$-jet of $M$ and denoted by $j_{\mathrm{P}}^{r}(F)$ if this class contains a mapping $F: R \rightarrow M$ such that $F(0)=\mathrm{P}$. The point $\mathrm{P}$ is called the target of the $r$-jet $j_{\mathrm{P}}^{r}(F)$. In the sequel, we shall restrict ourselves to the case $r=1$ or $r=2$.

If we denote by $T_{2}(M)$ the set of all 2-jets of $M$ and topologize $T_{2}(M)$ in the natural way, the space $T_{2}(M)$ has the natural bundle structure over $M$, its bundle projection $\pi_{2}: T_{2}(M) \rightarrow M$ being defined by $\pi_{2}\left(j_{\mathrm{P}}^{2}(F)\right)=\mathrm{P}$. The space $T_{2}(M)$ is called the tangent bundle of order 2 over $M$.

The set $T_{1}(M)$ of all 1 -jets of $M$ is nothing but the tangent bundle of $M$, if $T_{1}(M)$ is naturally topologized. The bundle projection $\pi_{1}: T_{1}(M) \rightarrow M$ of $T_{1}(M)$ is defined by $\pi_{1}\left(j_{\mathrm{P}}^{1}(F)\right)=\mathrm{P}$. Each 1 -jet of $M$ is called a tangent vector of $M$. If we introduce a mapping $\pi_{12}: T_{2}(M) \rightarrow T_{1}(M)$ by $\pi_{12}\left(j_{\mathrm{P}}^{2}(F)\right)=j_{\mathrm{P}}^{1}(F), F: R \rightarrow M$ being an arbitrary differentiable mapping such that $F(0)=\mathrm{P}$, then $T_{2}(M)$ has a bundle structure over $T_{1}(M)$ with bundle projection $\pi_{12}$. It is easily verified that the relation

$$
\pi_{2}=\pi_{1} \circ \pi_{12}
$$

holds.

Let $U$ be a coordinate neighborhood of $M$ and $\left(x^{h}\right)$ certain coordinates defined in $U$. We call the set $\left(U,\left(x^{h}\right)\right)$ simply a coordinate neighborhood of $M$. If we take an arbitrary 2-jet $j_{\mathrm{P}}^{2}(F)$ belonging to $\pi_{2}^{-1}(U)$ and put

2) Manifolds, mappings and objects we discuss are assumed to be differentiable and of class $C^{\infty}$. Manifolds under consideration are supposed to be connected.

3) The indices $h, i, j, k, \cdots, m, t, s$ run over the range $\{1,2, \cdots, n\}$ and the so-called Einstein's summation convention is used with respect to this system of indices. 


$$
y^{h}=\frac{d F^{h}(0)}{d t}, \quad z^{h}=\frac{d^{2} F^{h}(0)}{d t^{2}}
$$

then we see from (1.1) that the 2-jet $j_{\mathrm{P}}^{2}(F)$ is expressed in a unique way by the set $\left(x^{h}, y^{h}, z^{h}\right)$, where $x^{h}$ are the coordinates of the target $\mathrm{P}$ in $\left(U,\left(x^{h}\right)\right)$. Thus a system of coordinates $\left(x^{h}, y^{h}, z^{h}\right)$ is introduced in the open set $\pi_{2}^{-1}(U)$ of $T_{2}(M)$. We call $\left(x^{h}, y^{h}, z^{h}\right)$ the coordinates induced in $\pi_{2}^{-1}(U)$ from $\left(U,\left(x^{h}\right)\right)$, or, simply the induced coordinates in $\pi_{2}^{-1}(U)$. On putting

$$
\xi^{h}=x^{h}, \quad \xi^{\bar{h}}=y^{h}, \quad \xi^{\overline{\bar{h}}}=z^{h},
$$

we denote the induced coordinates $\left(x^{h}, y^{h}, z^{h}\right)$ by $\left(\xi^{A}\right)$ in $\pi_{2}{ }^{-1}(U) .^{4)}$

Let $\left(U,\left(x^{h}\right)\right)$ and $\left(U^{\prime},\left(x^{h^{\prime}}\right)\right)$ be two intersecting coordinate neighborhoods of $M$. Let $\left(\xi^{A}\right)=\left(x^{h}, y^{h}, z^{h}\right)$ and $\left(\xi^{A^{\prime}}\right)=\left(x^{h^{\prime}}, y^{h^{\prime}}, z^{h^{\prime}}\right)$ be the coordinates induced respectively from $\left(U,\left(x^{h}\right)\right)$ and $\left(U^{\prime},\left(x^{h^{\prime}}\right)\right)$. Then, denoting by $x^{h^{\prime}}=x^{h^{\prime}}\left(x^{i}\right)$ the coordinate transformation in $U \cap U^{\prime}$, the transformation of the induced coordinates in $\pi_{2}^{-1}\left(U \cap U^{\prime}\right)$ is given by

$$
x^{h^{\prime}}=x^{h^{\prime}}\left(x^{i}\right), \quad y^{h^{\prime}}=\frac{\partial x^{h^{\prime}}}{\partial x^{h}} y^{h}, \quad z^{h^{\prime}}=\frac{\partial x^{h^{\prime}}}{\partial x^{h}} z^{h}+\frac{\partial^{2} x^{h^{\prime}}}{\partial x^{j} \partial x^{2}} y^{\jmath} y^{\imath},
$$

and its Jacobian matrix by

$$
\left(\begin{array}{ccc}
\frac{\partial x^{h^{\prime}}}{\partial x^{h}} & 0 & 0 \\
\frac{\partial^{2} x^{h^{\prime}}}{\partial x^{i} \partial x^{s}} y^{s} & \frac{\partial x^{i^{\prime}}}{\partial x^{2}} & 0 \\
\frac{\partial^{2} x^{h^{\prime}}}{\partial x^{j} \partial x^{s}} z^{s}+\frac{\partial^{s} x^{h^{\prime}}}{\partial x^{j} \partial x^{t} \partial x^{s}} y^{t} y^{s} & 2 \frac{\partial^{2} x^{i^{\prime}}}{\partial x^{j} \partial x^{s}} y^{s} & \frac{\partial x^{j^{\prime}}}{\partial x^{j}}
\end{array}\right)
$$

Let $\varphi: M \rightarrow M$ be a differentiable transformation. The correspondence $j_{\mathrm{P}}^{2}(F)$ $\rightarrow j_{\varphi(\mathrm{P})}^{2}(\varphi \circ F), j_{\mathrm{P}}^{2}(F) \in T_{2}(M)$ determines a differentiable transformation $\varphi^{*}: T_{2}(M)$ $\rightarrow T_{2}(M)$, called the transformation induced in $T_{2}(M)$ from $\varphi$. If we take a point $\mathrm{P}$ belonging to a coordinate neighborhood $\left(U,\left(x^{h}\right)\right)$, and, if we suppose that the point $\varphi(\mathrm{P})$ belongs to a coordinate neighborhood $\left(U^{\prime},\left(x^{h^{\prime}}\right)\right)$, then we can express $\varphi$ locally by equations

$$
x^{h^{\prime}}=\varphi^{h^{\prime}}\left(x^{h}\right),
$$

$\varphi^{h^{\prime}}\left(x^{h}\right)$ being $n$ differentiable functions of the variables $x^{h}$ such that $\left|\partial \varphi^{h^{\prime}} / \partial x^{h}\right| \neq 0$, where $\left(x^{h}\right)$ are the coordinates of $\mathrm{P}$ in $\left(U,\left(x^{h}\right)\right)$ and $\left(x^{h^{\prime}}\right)$ those of $\varphi(\mathrm{P})$ in $\left(U^{\prime},\left(x^{h^{\prime}}\right)\right)$. Then the induced transformation $\varphi^{*}$ is expresssd locally by equations of the form

4) The indices $A, B, C, D, E$ run over the symbols $\{1,2, \cdots, n ; \overline{1}, \overline{2}, \cdots, \bar{n} ; \overline{\overline{1}}, \overline{\overline{2}}, \cdots, \overline{\bar{n}}\}$ and the so-called Einste!n's summation convention is used with respect to this system of indices, 
(1. 8)

$$
x^{h^{\prime}}=\varphi^{h^{\prime}}\left(x^{h}\right), \quad y^{h^{\prime}}=\frac{\partial \varphi^{h^{\prime}}}{\partial x^{h}} y^{h},
$$

$$
z^{h^{\prime}}=\frac{\partial \varphi^{h^{\prime}}}{\partial x^{h}} z^{h}+\frac{\partial^{2} \varphi^{h^{\prime}}}{\partial x^{j} \partial x^{2}} y^{\jmath} y^{2}
$$

where $\left(x^{h}, y^{h}, z^{h}\right)$ are the induced coordinates of $j_{\mathrm{P}}^{2}(F)$ in $\pi_{2}^{-1}(U)$ and $\left(x^{h^{\prime}}, y^{h^{\prime}}, z^{h^{\prime}}\right)$ those of $\varphi^{*}\left(j_{\mathrm{P}}^{2}(F)\right)$ in $\pi_{2}^{-1}\left(U^{\prime}\right)$.

Let $X$ be an infinitesimal transformation (a vector field) in $M$. Then, taking account of (1.8), we see easily that there naturally corresponds an infinitesimal transformation $\tilde{X}$ in $T_{2}(M)$ having components of the form

$$
\tilde{X}^{h}=X^{h}, \quad \tilde{X}^{\bar{n}}=y^{i} \partial_{i} X^{h}, \quad \tilde{X}^{\overline{\bar{h}}}=z^{i} \partial_{i} X^{h}+y^{\jmath} y^{i} \partial_{j} \partial_{i} X^{h}
$$

in $\pi_{2}^{-1}(U)$, the functions $X^{h}$ being the components of $X$ in $\left(U,\left(x^{h}\right)\right)$ and $\partial_{i}$ denoting the operator

$$
\partial_{i}=\frac{\partial}{\partial x^{2}}
$$

Hence we have the relation

$$
(\exp (t X))^{*}=\exp (t \tilde{X}) \quad(t \in R)
$$

whenever $\exp (t X)$ is defined.

If we put $Y=f X, f$ and $X$ being respectively a function and a vector field in $\mathrm{M}$, then we find in $T_{2}(M)$

$$
\tilde{Y}=\tilde{f} \tilde{X}+2 \tilde{g} \tilde{U}+\tilde{h} \tilde{V},
$$

$\tilde{X}$ and $\tilde{Y}$ being constructed by (1.9) respectively from $X$ and $Y$, where $\tilde{U}$ and $\tilde{V}$ are vector fields having respectively components of the form

$$
\tilde{U}: \quad \tilde{U}^{n}=0, \quad \tilde{U}^{\bar{n}}=\frac{1}{2} y^{i} \partial_{i} X^{n}, \quad \tilde{U}^{\bar{h}}=X^{n} ;
$$

$$
\tilde{V}: \quad \tilde{V}^{h}=0, \quad \tilde{V}^{\bar{n}}=0, \quad \tilde{V}^{\overline{\bar{h}}}=X^{h}
$$

in $\pi_{2}^{-1}(U)$ and $\tilde{f}=f_{\circ} \pi_{12}, \quad \tilde{g}=y^{i} \partial_{i} f, \tilde{h}=z^{i} \partial_{i} f+y^{j} y^{i} \partial_{\jmath} \partial_{i} f$ with respect to the induced coordinates $\left(x^{h}, y^{h}, z^{h}\right)$ in $\pi_{2}^{-1}(U)$. Therefore, given a vector field $X$ in $M$, we obtain in $T_{2}(M)$ three vector fields $\tilde{X}, \tilde{U}$, and $\tilde{V}$ defined by (1.9), (1.11) and (1.12) respectively.

\section{Notations.}

We list below notations used frequently in this paper.

1. $I_{s}^{r}(M)$ is the space of all tensor fields of type $(r, s)$, i.e., of contravariant degree $r$ and covariant degree $s$, in a differentiable manifold $M$. An element of 
$\mathscr{I}_{0}^{0}(M)$ is a function in $M$, an element of $\mathscr{I}_{0}^{1}(M)$ is a vector field in $M$, and an element of $\mathscr{I}_{1}^{0}(M)$ is a 1 -form in $M$.

2.

$$
\mathscr{I}(M)=\sum_{r, s} \mathscr{I}_{s}^{r}(M)
$$

3. $\Lambda_{*}(M)$ is the space of all differential forms in $M . \Lambda_{s}$ is the space of all $s$ forms in $M$.

$$
\Lambda_{*}(M)=\sum_{s} \Lambda_{s}(M), \quad \Lambda_{s}(M)=\Lambda_{*}(M) \cap \mathscr{I}_{s}^{0}(M) .
$$

4. A mapping $\varphi: \mathscr{I}(M) \rightarrow \mathscr{I}\left(M^{\prime}\right)$ is said to be linear if we have $\varphi(a S+b T)$ $=a \varphi(S)+b \varphi(T)$ for any element $S, T \in \mathscr{I}(M)$, where $a$ and $b$ are constants.

\section{$\S 2$. Lifts of functions.}

Lifts of functions. Let $f$ be a function in $M$. Then $f$ is a mapping $f: M \rightarrow R$ and it gives a mapping $f \circ F: R \rightarrow R$. For the given function $f$ a 2 -jet $j_{a}^{2}(f \circ F)$ of $R$ is completely determined by giving a 2 -jet $j_{\mathrm{P}}^{2}(F), F$ being a mapping $F: R \rightarrow M$ such that $\mathrm{P}=F(0)$ and $a=f(\mathrm{P})$. Thus, if we put $f^{\sharp}\left(j_{\mathrm{P}}^{2}(F)\right)=j_{a}^{2}(f \circ F)$, there exists a mapping $f^{\sharp}: T_{2}(M) \rightarrow T_{2}(R)$ corresponding to $f$. On the other hand, any element $\tau$ of $T_{2}(R)$ can be expressed canonically by a set $\left(A^{0}(\tau), A^{\mathrm{I}}(\tau), A^{\mathrm{II}}(\tau)\right)$ of three numbers, which are the induced coordinates of $\tau$ in $T_{2}(R)$, because $R$ is covered naturally by only one coordinate neighborhood $R$ itself. Therefore, for a function $f$ given in $M$, there corresponds in $T_{2}(M)$ three functions $f^{0}, f^{I}$ and $f^{I I}$ respectively defined by

$$
f^{0}(\sigma)=A^{0}\left(f^{\sharp}(\sigma)\right), \quad f^{\mathrm{I}}(\sigma)=A^{\mathrm{I}}\left(f^{\sharp}(\sigma)\right), \quad f^{\mathrm{II}}(\sigma)=A^{\mathrm{II}}\left(f^{\sharp}(\sigma)\right),
$$

$\sigma$ being an arbitrary element of $T_{2}(M)$. The three functions $f^{0}, f^{\text {I }}$ and $f^{\text {II }}$ thus defined in $T_{2}(M)$ is called respectively the $0-t h$, the 1 st and the 2 nd lifts of $f$. A function $f$ in $M$ is constant if and only if one of its lifts $f^{\mathrm{I}}$ and $f^{\text {II }}$ vanishes identically in $T_{2}(M)$. A function $f$ in $M$ vanishes identically if and only if its lift $f^{0}$ does so in $T_{2}(M)$.

The lifts $f^{0}, f^{\mathrm{I}}$ and $f^{\mathrm{II}}$ of a function $f$ in $M$ expressed by $f\left(x^{h}\right)$ in $\left(U,\left(x^{h}\right)\right)$ are represented respectively as

$$
f^{0}: f\left(x^{h}\right), \quad f^{\mathrm{I}}: y^{i} \partial_{i} f\left(x^{h}\right), \quad f^{\mathrm{II}}: z^{i} \partial_{i} f\left(x^{h}\right)+y^{\jmath} y^{i} \partial_{j} \partial_{i} f\left(x^{h}\right)
$$

with respect to the induced coordinates $\left(\xi^{A}\right)=\left(x^{h}, y^{h}, z^{h}\right)$ in $\pi_{2}^{-1}(U)$. We note here that $f^{0}$ has in $\pi_{2}^{-1}(U)$ the same local representation as $f$ has in $\left(U,\left(x^{h}\right)\right)$.

Taking account of (2.2), we find

$$
f^{0}=f \circ \pi_{2}=\left(f^{V}\right) \circ \pi_{12}, \quad f^{\mathrm{I}}=\left(f^{C}\right) \circ \pi_{12}
$$

for $f \in \mathscr{I}_{0}^{0}(M)$, where the functions $f^{V}$ and $f^{C}$ defined in $T_{1}(M)$ are respectively the vertical and the complete lifts of $f$ in the sense of [14] and [15]. As consequences 
of (2.2), we find the following formulas:

(2. 4)

$$
\begin{gathered}
(f g)^{0}=g^{0} f^{0}, \quad(f g)^{\mathrm{I}}=f^{\mathrm{I}} g^{0}+f^{0} g^{\mathrm{I}}, \\
(f g)^{\mathrm{II}}=f^{\mathrm{II}} g^{0}+2 f^{\mathrm{I}} g^{\mathrm{I}}+f^{0} g^{\mathrm{II}}
\end{gathered}
$$

for $g, f \in \mathscr{I}_{0}^{0}(M)$.

REMARK. Let $\tilde{X}$ be a vector field in $T_{2}(M)$. Then $\tilde{X}$ vanishes identically in $T_{2}(M)$ if we have $\tilde{X} f^{\mathrm{II}}=0$ for any function $f$ in $M$. In fact, if we take account of (2.2) and denote by $\left(\tilde{X}^{A}\right)=\left(\tilde{X}^{h}, \tilde{X}^{\tilde{h}}, \tilde{X}^{\bar{h}}\right)$ the components of $\tilde{X}$ with respect to the induced coordinates $\left(\xi^{A}\right)=\left(x^{h}, y^{h}, z^{h}\right)$, we see that the condition $\tilde{X} f^{I I}=0$ is expressed as

$$
\tilde{X}^{k}\left(z^{i} \partial_{k} \partial_{i} f+y^{\jmath} y^{i} \partial_{k} \partial_{j} \partial_{i} f\right)+2 \tilde{X}^{\bar{k}} y^{i} \partial_{k} \partial_{i} f+\tilde{X}^{\overline{\bar{k}}} \partial_{k} f=0
$$

Thus, if we have $\tilde{X} f^{\mathrm{II}}=0$ for any element $f$ of $\mathscr{I}_{0}^{0}(M)$, we find $\tilde{X}^{h}=\tilde{X}^{\bar{h}}=\tilde{X}^{\overline{\bar{h}}}=0$ by virtue of the continuity of $\tilde{X}$. Consequently, a vector field $\tilde{X}$ in $T_{2}(M)$ is completely determined by giving the values of $\tilde{X} f^{\text {II }}, f$ being arbitrary elements of $\mathscr{I}_{0}^{0}(M)$. In the sequel, this remark will be useful in determining values of vector fields given in $T_{2}(M)$.

$V$ ector fields $A$ and $B$. We now consider in each $\pi_{2}^{-1}(U)$ two local vector fields $A$ and $B$ respectively with components of the form

$$
A:\left(\begin{array}{c}
0 \\
0 \\
y^{h}
\end{array}\right), \quad B:\left(\begin{array}{c}
0 \\
\frac{1}{2} y^{h} \\
z^{h}
\end{array}\right)
$$

with respect to the induced coordinates $\left(\xi^{A}\right),\left(U,\left(x^{h}\right)\right)$ being an arbitrary coordinate neighborhood of $M$. Taking account of (1.5) and (1.6), we can easily verify that both of the local vector fields $A$ and $B$ thus introduced determine respectively global vector fields in $T_{2}(M)$, which are also denoted by $A$ and $B$ respectively. We now obtain the following formulas:

$$
A f^{0}=0, \quad A f^{\mathrm{I}}=0, \quad A f^{\mathrm{II}}=f^{\mathrm{I}},
$$

$$
B f^{0}=0, \quad B f^{\mathrm{I}}=\frac{1}{2} f^{\mathrm{I}}, \quad B f^{\mathrm{II}}=f^{\mathrm{II}}
$$

for $f \in \mathscr{I}_{0}^{0}(M)$ and

$$
[A, B]=\frac{1}{2} A
$$

by virtue of (2.2) and (2.5). 


\section{$\S 3$. Lifts of vector fields, 1-forms and derivations.}

Lifts of vector fields. Let $X$ be a vector field in $M$. We introduce in each $\pi_{2}^{-1}(U)$ three local vector fields $X^{0}, X^{I}$ and $X^{I I}$ having respective components of the form

$$
X^{0}=\left(\begin{array}{c}
0 \\
0 \\
X^{h}
\end{array}\right), \quad X^{\mathrm{I}}=\left(\begin{array}{c}
0 \\
\frac{1}{2} X^{h} \\
y^{i} \partial_{i} X^{h}
\end{array}\right), \quad X^{\mathrm{II}}=\left(\begin{array}{c}
X^{h} \\
y^{i} \partial_{i} X^{h} \\
y^{i} \partial_{i} X^{h}+y^{j} y^{i} \partial_{j} \partial_{i} X^{h}
\end{array}\right)
$$

with respect to the induced coordinates $\left(\xi^{A}\right)$, where $X^{h}$ denote the components of $X$ in $\left(U,\left(x^{h}\right)\right.$ ) (Cf. (1.9), (1.11) and (1.12)). If we take account of (1.5), (1.6) and the transformation law $X^{h^{\prime}}=\left(\partial x^{h^{\prime}} / \partial x^{h}\right) X^{h}$ of the components of $X$, then we see that the local vector fields $X^{0}, X^{\mathrm{I}}$ and $X^{\mathrm{II}}$ above determine respectively global vector fields in $T_{2}(M)$, which are also denoted by $X^{0}, X^{I}$ and $X^{I I}$ respectively. The vector fields $X^{0}, X^{\mathrm{I}}$ and $X^{\mathrm{II}}$ in $T_{2}(M)$ are called respectively the $0-t h$, the $1 s t$ and the 2 nd lifts of $X$. We find

$$
\pi_{12}\left(X^{0}\right)=0, \quad \pi_{12}\left(X^{\mathrm{I}}\right)=\frac{1}{2} X^{V}, \quad \pi_{12}\left(X^{\mathrm{II}}\right)=X^{C}
$$

for $X \in \mathscr{I}_{0}^{1}(M)$ because of (3.1), $\pi_{12}$ denoting the differential mapping of the projection $\pi_{12}: T_{2}(M) \rightarrow T_{1}(M)$, where the vector fields $X^{v}$ and $X^{C}$ defined in $T_{1}(M)$ denote respectively the vertical and the complete lifts of $X$ in the sense of [14] and [15]. According to (3.1), a vector field $X$ in $M$ vanishes identically if and only if one of $X^{0}, X^{\mathrm{I}}$ and $X^{\mathrm{II}}$ does so in $T_{2}(M)$.

Taking account of (3.1), we find the following formulas:

$$
(f X)^{0}=f^{0} X^{0}, \quad(f X)^{\mathrm{I}}=f^{\mathrm{I}} X^{0}+f^{0} X^{\mathrm{I}},
$$

$$
(f X)^{\mathrm{II}}=f^{\mathrm{II}} X^{0}+2 f^{\mathrm{I}} X^{\mathrm{I}}+f^{0} X^{\mathrm{II}}
$$

for $f \in \mathscr{I}_{0}^{0}(M), X \in \mathscr{I}_{0}^{1}(M)$. As immediate consequences of (2.2) and (3.1), we have the following formulas:

$$
X^{0} f^{0}=0, \quad X^{0} f^{\mathrm{I}}=0, \quad X^{0} f^{\mathrm{II}}=(X f)^{0},
$$

$$
\begin{aligned}
X^{\mathrm{I}} f^{0}=0, & X^{\mathrm{I}} f^{\mathrm{I}}=\frac{1}{2}(X f)^{0}, & X^{\mathrm{I}} f^{\mathrm{II}}=(X f)^{\mathrm{I}}, \\
X^{\mathrm{II}} f^{0}=(X f)^{0}, & X^{\mathrm{II}} f^{\mathrm{I}}=(X f)^{\mathrm{I}}, & X^{\mathrm{II}} f^{\mathrm{II}}=(X f)^{\mathrm{II}}
\end{aligned}
$$

for $f \in \mathscr{I}_{0}^{0}(M), X \in \mathscr{I}_{0}^{1}(M)$.

Lifts of 1-forms. Let $\omega$ be a 1-form in $M$. We introduce in each $\pi_{2}^{-1}(U)$ three local 1 -forms $\omega^{0}$, $\omega^{\mathrm{I}}$ and $\omega^{\mathrm{II}}$ having respective components of the form 


$$
\begin{aligned}
\omega^{0}: & \left(\omega_{i}, 0,0\right), \\
\omega^{\mathrm{I}}: & \left(y^{k} \partial_{k} \omega_{i}, \omega_{i}, 0\right), \\
\omega^{\mathrm{I}}: & \left(z^{k} \partial_{k} \omega_{i}+y^{k} y^{j} \partial_{k} \partial_{j} \omega_{i}, 2 y^{j} \partial_{j} \omega_{i}, \omega_{i}\right)
\end{aligned}
$$

with respect to the induced coordinates $\left(\xi^{A}\right)$, where $\omega_{i}$ denote the components of $\omega$ in $\left(U,\left(x^{h}\right)\right)$. Taking account of (1.5), (1.6) and the transformation law $\omega_{i}=\left(\partial x^{i} / \partial x^{i^{\prime}}\right) \omega_{i}$ of components of $\omega$, we can easily verify that the local 1 -forms $\omega^{0}$, $\omega^{\mathrm{I}}$ and $\omega^{\mathrm{II}}$ above determine respectively global 1-forms in $T_{2}(M)$, which are also denoted respectively by $\omega^{0}, \omega^{\mathrm{I}}$ and $\omega^{\mathrm{II}}$. These 1 -forms $\omega^{0}, \omega^{\mathrm{I}}$ and $\omega^{\mathrm{II}}$ are respectively called the 0 -th, the $1 s t$ and the $2 n d$ lifts of $\omega$. From (3.5) we find

$$
\omega^{0}=\omega \circ \pi_{2}=\omega^{V} \circ \pi_{12}, \quad \omega^{\mathrm{I}}=\omega^{C} \circ \pi_{12}
$$

for $\omega \in \mathscr{I}_{0}^{1}(M)$, where the 1 -forms $\omega^{V}$ and $\omega^{C}$ defined in $T_{2}(M)$ are respectively the vertical and the complete lifts of $\omega$ in the sense of [14] and [15]. According to (3. 5), a 1-form $\omega$ vanishes identically in $M$ if and only if one of $\omega^{0}$, $\omega^{\mathrm{I}}$ and $\omega^{\mathrm{II}}$ does so in $T_{2}(M)$.

Taking account of (3.5), we obtain the formulas

$$
\begin{gathered}
(f \omega)^{0}=f^{0} \omega^{0}, \quad(f \omega)^{\mathrm{I}}=f^{\mathrm{I}} \omega^{0}+f^{0} \omega^{\mathrm{I}}, \\
(f \omega)^{\mathrm{II}}=f^{\mathrm{II}} \omega^{0}+2 f^{\mathrm{I}} \omega^{\mathrm{I}}+f^{0} \omega^{\mathrm{II}}
\end{gathered}
$$

for $f \in \mathscr{I}_{0}^{0}(M), \omega \in \mathscr{I}_{1}^{\mathrm{n}}(M)$. As immediate consequences of (3.1) and (3.5), we find the following formulas:

$$
\begin{array}{rlrl}
\omega^{0}\left(X^{0}\right) & =0, & \omega^{0}\left(X^{\mathrm{I}}\right)=0 & \omega^{0}\left(X^{\mathrm{II}}\right)=(\omega(X))^{0}, \\
\omega^{\mathrm{I}}\left(X^{0}\right)=0, & \omega^{\mathrm{I}}\left(X^{\mathrm{I}}\right)=\frac{1}{2}(\omega(X))^{0}, & \omega^{\mathrm{I}}\left(X^{\mathrm{II}}\right)=(\omega(X))^{\mathrm{I}}, \\
\omega^{\mathrm{II}}\left(X^{0}\right)=(\omega(X))^{0}, & \omega^{\mathrm{II}}\left(X^{\mathrm{I}}\right)=(\omega(X))^{\mathrm{I}}, & \omega^{\mathrm{II}}\left(X^{\mathrm{II}}\right)=(\omega(X))^{\mathrm{II}}
\end{array}
$$

for $X \in \mathscr{I}_{0}^{1}(M), \omega \in \mathscr{I}_{1}^{0}(M)$.

Formulas. We have here the following formulas:

$$
\begin{aligned}
{\left[X^{0}, Y^{0}\right] } & =0, & {\left[X^{\mathrm{I}}, Y^{\mathrm{I}}\right] } & =\frac{1}{2}[X, Y]^{0}, \\
{\left[X^{\mathrm{I}}, Y^{0}\right] } & =0, & {\left[X^{\mathrm{II}}, Y^{\mathrm{I}}\right] } & =[X, Y]^{\mathrm{I}}, \\
{\left[X^{\mathrm{II}}, Y^{0}\right] } & =[X, Y]^{0}, & {\left[X^{\mathrm{II}}, Y^{\mathrm{II}}\right] } & =[X, Y]^{\mathrm{II}}
\end{aligned}
$$

for $X, Y \in I_{0}^{1}(M)$. In fact, taking account of (3.4), we have

$$
\begin{aligned}
{\left[X^{\mathrm{II}}, Y^{\mathrm{I}}\right] f^{\mathrm{II}} } & =X^{\mathrm{II}}\left(Y^{\mathrm{I}} f^{\mathrm{II}}\right)-Y^{\mathrm{I}}\left(X^{\mathrm{II}} f^{\mathrm{II}}\right)=\left(X(Y f)^{\mathrm{I}}-(Y(X f))^{\mathrm{I}}\right. \\
& =([X, Y] f)^{\mathrm{I}}=[X, Y]^{\mathrm{I}} f^{\mathrm{II}},
\end{aligned}
$$




$$
\left[X^{\mathrm{II}}, Y^{\mathrm{II}}\right] f^{\mathrm{II}}=([X, Y] f)^{\mathrm{II}}=[X, Y]^{\mathrm{II}} f^{\mathrm{II}}
$$

for any element $f$ of $\mathscr{I}_{0}^{0}(M)$. Therefore, if we take account of the Remark stated in $\S 2$, we obtain $\left[X^{\mathrm{II}}, Y^{\mathrm{I}}\right]=[X, Y]^{\mathrm{I}}$ and $\left[X^{\mathrm{II}}, Y^{\mathrm{II}}\right]=[X, Y]^{\mathrm{II}}$. Applying similar devices, we can prove the other formulas given in (3.9).

The correspondences $X \rightarrow X^{0}, X \rightarrow X^{\mathrm{I}}$ and $X \rightarrow X^{\text {II }}\left(X \in \mathscr{I}_{0}^{1}(M)\right)$ determine respectively one-to-one linear mappings of $\mathscr{I}_{0}^{1}(M)$ into $\mathfrak{I}_{0}^{1}\left(T_{2}(M)\right)$. We have, from the last formulas given in (3.9),

Proposition 3. 1. The correspondence $X \rightarrow X^{11}\left(X \in \mathscr{I}_{0}^{1}(M)\right)$ determines an isomorphism of the Lie algebra $\mathscr{I}_{0}^{1}(M)$ into the Lie algebra $\mathscr{I}_{0}^{1}\left(T_{2}(M)\right)$.

According to (3.1) and (3. 5), we find in each neighborhood $\pi_{2}^{-1}(U)$ the formulas

$$
\left(\frac{\partial}{\partial x^{2}}\right)^{0}=\frac{\partial}{\partial z^{2}}, \quad\left(\frac{\partial}{\partial x^{2}}\right)^{\mathrm{I}}=\frac{1}{2}-\frac{\partial}{\partial y^{2}}, \quad\left(\frac{\partial}{\partial x^{2}}\right)^{\mathrm{II}}=\frac{\partial}{\partial x^{2}} ;
$$

$$
\left(d x^{h}\right)^{0}=d x^{h}, \quad\left(d x^{h}\right)^{\mathrm{I}}=d y^{h}, \quad\left(d x^{h}\right)^{\mathrm{II}}=d z^{h}
$$

with respect to the induced coordinateg $\left(\xi^{A}\right)=\left(x^{h}, y^{h}, z^{h}\right)$, where $\left(U,\left(x^{h}\right)\right)$ is a coordinate neighborhood of $M$.

REMARK. If we take account of (3.1) and (3.5), we see that a tensor field $K$, say, of type $(1,2)$ in $T_{2}(M)$ is completely determined by giving values $K\left(X^{\mathrm{II}}, Y^{\mathrm{II}}, \omega^{\mathrm{II}}\right)$, $X$ and $Y$ being arbitray elements of $\mathscr{I}_{0}^{1}(M)$ and $\omega$ an arbitrary element of $\mathscr{I}_{1}^{0}(M)$.

Lifts of derivations. In this paper we mean by a derivation in $M$ a linear mapping $D: \mathscr{I}(M) \rightarrow \mathscr{I}(M)$ satisfying the conditions:
(a) $D: \mathscr{I}_{s}^{r}(M) \rightarrow \mathscr{I}_{s}^{r}(M)$,

(b) $D(S \otimes T)=(D S) \otimes T+S \otimes(D T) \quad$ for $S, T \in \mathscr{I}(M)$,

(c) $D I=0$,

where $I$ denotes the identity tensor field of type $(1,1)$ in $M$.

For a given derivation $D$ in $M$, there exists a vector field $P$ in $M$ such that

$$
P f=D f,
$$

$f$ being an arbitrary element of $\mathscr{I}_{0}^{0}(M)$. In each coordinate neighborhood $\left(U,\left(x^{h}\right)\right)$ of $M$, taking account of (3.11, a), we can put

$$
D\left(\frac{\partial}{\partial x^{2}}\right)=Q_{i}{ }^{h} \frac{\partial}{\partial x^{h}}
$$

$Q_{i}{ }^{h}$ being certain functions in $U$. Thus, taking account of $(3.11, \mathrm{~b}),(3.12)$ and 
(3. 13), we obtain

$$
D\left(X^{h} \frac{\partial}{\partial x^{h}}\right)=\left(P^{i} \partial_{i} X^{h}+Q_{i}{ }^{h} X^{i}\right) \frac{\partial}{\partial x^{h}}
$$

in $\left(U,\left(x^{h}\right)\right)$ for any element $X=X^{h}\left(\partial / \partial x^{h}\right)$ of $\mathscr{I}_{0}^{1}(M)$. That is to say, for any element $X$ of $\mathcal{I}_{0}^{1}(M), D X$ has components of the form

$$
(D X)^{h}=P^{i} \partial_{i} X^{h}+Q_{i}{ }^{h} X^{i}
$$

in $\left(U,\left(x^{h}\right)\right)$, if $X$ has components $X^{h}$ in $\left(U,\left(x^{h}\right)\right)$. According to (3.11), we have $D(\omega(X))=(D \omega)(X)+\omega(D X)$ for any element $X$ of $\mathscr{I}_{0}^{1}(M)$ and any element $\omega$ of

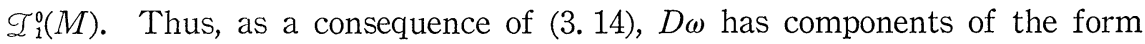

$$
(D \omega)_{i}=P^{j} \partial_{j} \omega_{i}-Q_{i}{ }^{h} \omega_{h} \quad \text { for } \quad \omega \in \mathscr{I}_{1}^{0}(M)
$$

in $\left(U,\left(x^{h}\right)\right)$, if $\omega$ has components $\omega_{i}$ in $\left(U,\left(x^{h}\right)\right)$. The set $\left(P^{h}, Q_{i}{ }^{h}\right)$ is called the components of the derivation $D$ in $\left(U,\left(x^{h}\right)\right)$.

We suppose that a derivation $D$ has components $\left(P^{h}, Q_{i}{ }^{h}\right)$ and $\left(P^{h^{\prime}}, Q_{i}{ }^{h^{\prime}}\right)$ respectively in $\left(U,\left(x^{h}\right)\right)$ and in $\left(U^{\prime},\left(x^{h^{\prime}}\right)\right)$. Then, as a consequence of (3.14) and the transformation law $X^{h^{\prime}}=\left(\partial x^{h^{\prime}} / \partial x^{h}\right) X^{h}$ of the components $X^{h}$ of $X$, we obtain the transformation law

$$
\begin{aligned}
P^{h^{\prime}} & =\frac{\partial x^{h^{\prime}}}{\partial x^{h}} P^{h}, \\
Q_{i^{\prime}}{ }^{\prime} & =\frac{\partial x^{h^{\prime}}}{\partial x^{h}}\left(\frac{\partial x^{2}}{\partial x^{i^{\prime}}} Q_{i}{ }^{h}+P^{\jmath} \frac{\partial x^{j^{\prime}}}{\partial x^{\jmath}} \frac{\partial_{2} x^{h}}{\partial x^{j^{\prime}} \partial x^{i^{\prime}}}\right)
\end{aligned}
$$

of the components of a derivation $D$ in $U \cap U^{\prime}$.

If we are given a derivation $D$ in $M$, we introduce in $\pi_{2}^{-1}(U)$ three local vector fields $D^{0}, D^{\mathrm{I}}$ and $D^{\text {II }}$ having components of the form

$$
D^{0}:\left(\begin{array}{c}
0 \\
0 \\
P^{h}
\end{array}\right), \quad D^{\mathrm{I}}:\left(\begin{array}{c}
0 \\
\frac{1}{2} P^{h} \\
-y^{2} Q_{i}{ }^{h}
\end{array}\right), \quad D^{\mathrm{II}:}\left(\begin{array}{c}
P^{h} \\
\frac{1}{2} y^{2}\left(\partial_{i} P^{h}-Q_{i}{ }^{h}\right) \\
-\left(z^{2} Q_{i}{ }^{h}+y^{j} y^{i} \partial_{j} Q_{i}{ }^{h}\right)
\end{array}\right)
$$

with respect to the induced coordinates $\left(\xi^{A}\right)$, where $\left(P^{h}, Q_{i}{ }^{h}\right)$ denote the components of the given derivation $D$ in $\left(U,\left(x^{h}\right)\right)$. Thus, taking account of (1.5), (1.6), (3.16) and (3.17), we see that all of the local vector fields $D^{0}, D^{\mathrm{I}}$ and $D^{\text {II }}$ above determine respectively global vector fields in $T_{2}(M)$, which are denoted also by $D^{0}, D^{\mathrm{I}}$ and $D^{\text {II }}$ respectively. These three vector fields $D^{0}, D^{\mathrm{I}}$ and $D^{\mathrm{II}}$ in $T_{2}(M)$ are called respectively the $0-t h$, the 1 st and the 2 nd lifts of the derivation $D$.

We now find for any derivation $D$ the following formulas: 


$$
\begin{aligned}
D^{0} f^{0}=0, & D^{\mathrm{I}} f^{0}=0, & D^{\mathrm{II}} f^{0}=(D f)^{0}, \\
D^{0} f^{\mathrm{I}}=0, & D^{\mathrm{I}} f^{\mathrm{I}}=\frac{1}{2}(D f)^{0}, & D^{\mathrm{II}} f^{\mathrm{I}}=\alpha(D d f), \\
D^{0} f^{\mathrm{II}}=(D f)^{0}, & D^{\mathrm{I}} f^{\mathrm{II}}=\alpha(D d f), & D^{\mathrm{II}} f^{\mathrm{II}}=\beta(D d f)
\end{aligned}
$$

for $f \in \mathscr{I}_{0}^{0}(M)$, where $\alpha \omega$ and $\beta \omega$ for any element $\omega$ of $\mathscr{I}_{1}^{0}(M)$ are functions in $T_{2}(M)$ having respectively local representations $\alpha \omega=y^{2} \omega_{i}$ and $\beta \omega=z^{2} \omega_{i}+y^{3} y^{i} \partial_{j} \omega_{i}$ in $\pi_{2}^{-1}(U)$ with respect to the induced coordinates $\left(\xi^{A}\right)$, the functions $\omega_{i}$ being the components of $\omega$ in $\left(U,\left(x^{h}\right)\right)$ (Cf. $\left.\S 5\right)$.

Lifts of Lie derivations. The Lie derivation $\mathcal{L}_{X}$ with respect to a vector field $X$ is a derivation having components of the form

$$
\mathcal{L}_{X}: \quad P^{h}=X^{h}, \quad Q_{i}{ }^{h}=-\partial_{i} X^{h},
$$

where $X^{h}$ denote the components of $X$. Thus, substituting (3.19) in (3.17), we have

Proposition 3. 2. The formulas

$$
\left(\mathcal{L}_{X}\right)^{0}=X^{0}, \quad\left(\mathcal{L}_{X}\right)^{\mathrm{I}}=X^{\mathrm{I}}, \quad\left(\mathcal{L}_{X}\right)^{\mathrm{II}}=X^{\mathrm{II}}
$$

hold for $X \in \mathscr{I}_{0}^{1}(M)$.

Lifts of covariant derivations. Let $\nabla$ be an affine connection in $M$. Then the covariant differentiation $\nabla_{X}$ with respect to a vector field $X$ is a derivation in $M$, which has components of the form

$$
\nabla_{X}: \quad P^{h}=X^{h}, \quad Q_{i}^{h}=X^{j} \Gamma_{j}^{h} \imath,
$$

$\Gamma_{j}{ }^{h} i$ denoting the coefficients of $\nabla$ and $X^{h}$ the components of $X$. The covariant derivative $\nabla_{X} Z$ has components of the form

$$
\left(\nabla_{X} Z\right)^{h}=X^{j}\left(\partial_{j} Z^{h}+\Gamma_{j}{ }^{h} Z^{i}\right)
$$

for any vector field $Z$ with components $Z^{h}$. Substituting (3.20) in (3.17), we see that the lifts $\left(\nabla_{X}\right)^{0},\left(\nabla_{X}\right)^{\mathrm{I}}$ and $\left(\nabla_{X}\right)^{\mathrm{II}}$ have respectively components of the form

$$
\left(\nabla_{X}\right)^{0}:\left(\begin{array}{c}
0 \\
0 \\
X^{h}
\end{array}\right), \quad\left(\nabla_{X}\right)^{\mathrm{I}}:\left(\begin{array}{c}
0 \\
\frac{1}{2} X^{h} \\
-X^{j} y^{\imath} \Gamma_{j}{ }^{h}{ }_{i}
\end{array}\right) \text {, }
$$

$$
\left(\nabla_{X}\right)^{\mathrm{II}}:\left(\begin{array}{c}
X^{h} \\
\frac{1}{2} y^{i}\left(\partial_{i} X^{h}-X^{j} \Gamma_{j}{ }^{h} i\right) \\
-\left(X^{\jmath} z^{i} \Gamma_{j}{ }^{h}{ }_{i}+y^{\jmath} y^{i} \partial_{j}\left(X^{k} \Gamma_{k}{ }^{h} \imath\right)\right.
\end{array}\right)
$$


for any element $X$ of $\mathscr{I}_{0}^{1}(M)$. Therefore we have, from (3.1) and (3.21),

Proposition 3. 3. The formulas

$$
\left(\nabla_{X}\right)^{0}=X^{0}, \quad\left(\nabla_{X}\right)^{\mathrm{I}}=X^{\mathrm{I}}-\alpha(\hat{\nabla} X), \quad\left(\nabla_{X}\right)^{\mathrm{II}}=X^{\mathrm{II}}-\beta(\hat{\nabla} X)
$$

hold for any element $X$ of $\mathscr{I}_{0}^{1}(M)$.

In Proposition 3. $3, \hat{V}$ is an affine connection in $M$ defined by

$$
\hat{\nabla}_{X} Y=\nabla_{Y} X+[X, Y] \quad \text { for } \quad X, Y \in \mathscr{L}_{0}^{1}(M) \text {, }
$$

and $\alpha F$ and $\beta F$ for any element $F$ of $\mathscr{I}_{1}^{1}(M)$ are vector fields in $T_{2}(M)$ having respectively components

$$
\alpha F:\left(\begin{array}{c}
0 \\
0 \\
y^{i} F_{i}^{h}
\end{array}\right), \quad \beta F:\left(\begin{array}{c}
0 \\
\frac{1}{2} y^{i} F_{i}{ }^{h} \\
z^{i} F_{i}^{h}+y^{\jmath} y^{i} \partial_{j} F_{i}^{h}
\end{array}\right)
$$

with respect to the induced coordinates $\left(\xi^{A}\right)$ in $\pi_{2}{ }^{-1}(U)$, the functions $F_{i}{ }^{h}$ being components of $F$ in $\left(U,\left(x^{h}\right)\right)$ (Cf. $\S 4$ or $\S 5$ ). We see easily that the affine connection $\hat{V}$ has coefficients $\hat{\Gamma}_{\jmath}{ }^{h}{ }_{\imath}=\Gamma_{\imath}{ }^{h}, \Gamma_{\jmath}{ }_{\imath}$ being the coefficients of $\nabla$. As an immediate consequence of Proposition 3.3, we have

Proposition 3.4. For any element $X$ of $\mathscr{I}_{0}^{1}(M)$

$$
\left(\nabla_{X}\right)^{\mathrm{I}}=X^{\mathrm{I}}, \quad\left(\nabla_{X}\right)^{\mathrm{II}}=X^{\mathrm{II}}
$$

hold if and only if $\hat{\nabla} X=0$.

Derivation determined by a tensor field of type $(1,1)$. When a derivation $D$ satisfies the condition $D f=0$ for $f \in \mathscr{I}_{0}^{0}(M), D$ determines an element $F$ of $\mathscr{I}_{1}^{1}(M)$ such that $D X=F X$ for any element $X$ of $\mathscr{I}_{0}^{1}(M)$. In such a case, we denote $D$ by $D_{F}$ and call it the derivation determined by a tensor field $F$ of type $(1,1)$. The derivation $D_{F}$ has components of the form

$$
D_{F}: \quad P^{h}=0, \quad Q_{i}^{h}=F_{i}^{h},
$$

$F_{i}^{h}$ being components of $F$. Substituting (3.23) in (3.17), we find

$$
\left(D_{F}\right)^{0}=0, \quad\left(D_{F}\right)^{\mathrm{I}}=-\alpha F, \quad\left(D_{F}\right)^{\mathrm{II}}=-\beta F,
$$

$\alpha F$ and $\beta F$ being defined by (3. 22). 


\section{$\S 4$. Lifts of tensor fields.}

Lifts of tensor fields. We have introduced in $\S 2$ and $\S 3$ three kinds of lifts for functions, vector fields and 1-forms given in $M$. The operations taking these lifts are linear mappings $\mathscr{I}_{0}^{0}(M) \rightarrow \mathscr{I}_{0}^{0}\left(T_{2}(M)\right), \mathscr{I}_{0}^{1}(M) \rightarrow \mathscr{I}_{0}^{1}\left(T_{2}(M)\right)$ and $\mathscr{I}_{1}^{0}(M) \rightarrow \mathscr{I}_{1}^{0}\left(T_{2}(M)\right)$ respectively. Thus we can now define for any element $K$ of $\mathscr{I}_{s}^{r}(M)$ its lifts $K^{0}$, $K^{\mathrm{I}}$ and $K^{\mathrm{II}}$, which are elements of $\mathscr{I}_{s}^{r}\left(T_{2}(M)\right)$, in such a way that the correspondence $K \rightarrow K^{0}, K \rightarrow K^{\mathrm{I}}$ and $K \rightarrow K^{\mathrm{II}}$ all define linear mappings $\mathscr{I}_{s}^{r}(M) \rightarrow \mathscr{I}_{s}^{r}\left(T_{2}(M)\right.$ ), which are characterized by the properties

$$
\begin{aligned}
(\mathrm{S} \otimes T)^{0} & =S^{0} \otimes T^{0}, \\
(\mathrm{~S} \otimes T)^{\mathrm{I}} & =S^{\mathrm{I}} \otimes T^{0}+S^{0} \otimes T^{\mathrm{I}}, \\
(\mathrm{S} \otimes T)^{\mathrm{II}} & =S^{\mathrm{II}} \otimes T^{0}+2 S^{\mathrm{I}} \otimes T^{\mathrm{I}}+S^{0} \otimes T^{\mathrm{II}}
\end{aligned}
$$

for $S, T \in \mathscr{I}(M)$. The conditions (4.1) are compatible with the conditions (2.4), (3. 3) and (3.7). The tensor fields $K^{0}, K^{\mathrm{I}}$ and $K^{\mathrm{II}}$ are called respectively the 0 - $t h$, the 1 st and the 2 nd lifts of $K$. We see that a tensor field $K$, not belonging to $I_{0}^{0}(M)$, vanishes identically in $M$ if and only if one of its lifts $K^{0}, K^{\mathrm{I}}$ and $K^{\mathrm{II}}$ does so in $T_{2}(M)$.

Linear mappings $\gamma_{x}$. Let $T$ be an element of $\mathscr{I}_{s}^{r}(M)(s \geqq 1)$. Then it is a correspondence

$$
T:\left(X_{1}, \cdots, X_{s}\right) \rightarrow T\left(X_{1}, \cdots, X_{s}\right) \in \mathscr{I}_{0}^{r}(M),
$$

$X_{1}, \cdots, X_{s}$ being arbitrary elements of $\mathscr{I}_{0}^{1}(M)$. If for an element $X$ of $\mathscr{I}_{0}^{1}(M)$ we define an element $\gamma_{x} T$ of $\mathscr{I}_{s-1}^{\prime}(M)$ by

$$
\left(\gamma_{X} T\right)\left(X_{2}, \cdots, X_{s}\right)=T\left(X, X_{2}, \cdots, X_{s}\right)
$$

$X_{2}, \cdots, X_{s}$ being arbitrary elements of $\mathscr{I}_{0}^{1}(M)$, then the correspondence $T \rightarrow \gamma_{X} T$ determines a mapping $\gamma_{X}: \mathscr{I}_{s}^{r}(M) \rightarrow \mathscr{I}_{s-1}^{r}(M)$ such that $\gamma_{X}(f T+g S)=f\left(\gamma_{X} T\right)+g\left(\gamma_{X} S\right)$ for $f, g \in \mathscr{I}_{0}^{0}(M)$ and $T, S \in \mathscr{I}_{s}^{r}(M)$. If $T$ has components of the form $T_{\imath_{1} 2_{2} \cdots l_{s}}{ }^{h_{1} \cdots h_{r}}$, then $\gamma_{X} T$ has the components $X^{k} T_{k v_{2} \cdots i_{s}}{ }^{h_{1} \cdots h_{r}}, X^{k}$ being components of $X$. We have the formula

$$
\gamma_{X_{s}} \cdots \gamma_{X_{1}} T=T\left(X_{1}, \cdots, X_{s}\right) \in \mathscr{I}_{0}^{r}(M)
$$

for any elements $X_{1}, \cdots, X_{s}$ of $\mathscr{I}_{0}^{1}(M)$.

We now have the following formulas:

$$
\begin{array}{lll}
\gamma_{X^{0}} K^{0}=0, & \gamma_{X^{\mathrm{I}}} K^{0}=0, & \gamma_{X^{1 I}} K^{0}=\left(\gamma_{X} K\right)^{0}, \\
\gamma_{X^{0}} K^{\mathrm{I}}=0, & \gamma_{X} K^{\mathrm{I}}=\frac{1}{2}\left(\gamma_{X} K\right)^{0}, & \gamma_{X} \mathrm{II} K^{\mathrm{I}}=\left(\gamma_{X} K\right)^{\mathrm{I}}, \\
\gamma_{X^{0}} K^{\mathrm{II}}=\left(\gamma_{X} K\right)^{0}, & \gamma_{X^{\mathrm{I}}} K^{\mathrm{II}}=\left(\gamma_{X} K\right)^{\mathrm{I}}, & \gamma_{X^{\mathrm{II}}} K^{\mathrm{II}}=\left(\gamma_{X} K\right)^{\mathrm{II}}
\end{array}
$$


for $X \in \mathscr{I}_{0}^{1}(M), K \in \mathscr{I}(M)$. In fact, if we suppose that $K=\omega \otimes S, \omega \in \mathscr{I}_{1}^{0}(M), S \in \mathscr{I}(M)$, then we have

$$
\begin{aligned}
\gamma_{X^{0}} K^{0} & =\gamma_{X^{0}}\left(\omega^{0} \otimes S^{0}\right)=\omega^{0}\left(X^{0}\right) S^{0}=0, \\
\gamma_{X^{\mathrm{I}}} K^{\mathrm{I}} & =\gamma_{X^{\mathrm{I}}}\left(\omega^{\mathrm{I}} \otimes S^{0}+\omega^{0} \otimes S^{\mathrm{I}}\right)=\frac{1}{2}(\omega(X))^{0} S^{0} \\
& =\frac{1}{2}\left(\gamma_{X}(\omega \otimes S)\right)^{0}=\frac{1}{2}\left(\gamma_{X} K\right)^{0}, \\
\gamma_{X}{ }^{1 \mathrm{II}} K^{\mathrm{II}} & =\gamma_{X}{ }^{\mathrm{II}}\left(\omega^{\mathrm{II}} \otimes S^{0}+2 \omega^{\mathrm{I}} \otimes S^{\mathrm{I}}+\omega^{0} \otimes S^{\mathrm{II}}\right) \\
& =\left((\omega(X))^{\mathrm{II}} S^{0}+2(\omega(X))^{\mathrm{I}} S^{\mathrm{I}}+(\omega(X))^{0} S^{\mathrm{II}}\right) \\
& =(\omega(X) S)^{\mathrm{II}}=\left(\gamma_{X}(\omega \otimes S)\right)^{\mathrm{II}}=\left(\gamma_{X} K\right)^{\mathrm{II}}
\end{aligned}
$$

by virtue of (3.8) and (4.1). Thus, according to $\gamma_{X}(f S+g T)=f \gamma_{X} S+g \gamma_{X} T$ for $S, T \in \mathscr{I}_{s}^{r}(M)$ and $f, g \in \mathscr{I}_{0}^{0}(M)$, we can prove these three formulas for any element $K$ of $\mathscr{I}(M)$. In a similar way, we can prove the other formulas given in (4. 2).

Lifts of differential forms. We now obtain the following formulas:

$$
\begin{gathered}
(\omega \wedge \pi)^{0}=\omega^{0} \wedge \pi^{0}, \quad(\omega \wedge \pi)^{\mathrm{I}}=\omega^{\mathrm{I}} \wedge \pi^{0}+\omega^{0} \wedge \pi^{\mathrm{I}}, \\
(\omega \wedge \pi)^{\mathrm{II}}=\omega^{\mathrm{II}} \wedge \pi^{0}+2 \omega^{\mathrm{I}} \wedge \pi^{\mathrm{I}}+\omega^{0} \wedge \pi^{\mathrm{II}}
\end{gathered}
$$

for $\omega, \pi \in \Lambda_{*}(M)$. Moreover we have the following formulas:

$$
\begin{aligned}
\omega^{0}\left(X^{\mathrm{II}}, Y^{\mathrm{II}}, \cdots, Z^{\mathrm{II}}\right) & =(\omega(X, Y, \cdots, Z))^{0}, \\
\omega^{\mathrm{I}}\left(X^{\mathrm{II}}, Y^{\mathrm{II}}, \cdots, Z^{\mathrm{II}}\right) & =(\omega(X, Y, \cdots, Z))^{\mathrm{I}}, \\
\omega^{\mathrm{II}}\left(X^{\mathrm{II}}, Y^{\mathrm{II}}, \cdots, Z^{\mathrm{II}}\right) & =(\omega(X, Y, \cdots, Z))^{\mathrm{II}}
\end{aligned}
$$

for $\omega \in \Lambda_{*}(M), X, Y, \cdots, Z$ being arbitrary element of $\mathscr{I}_{0}^{1}(M)$. The formulas (4.4) are immediate consequences of (4.2).

We obtain directly from (2.2) and (3.5)

$$
(d f)^{0}=d\left(f^{0}\right), \quad(d f)^{\mathrm{I}}=d\left(f^{\mathrm{I}}\right), \quad(d f)^{\mathrm{II}}=d\left(f^{\mathrm{II}}\right)
$$

for $f \in \mathscr{L}_{0}^{0}(M)$. We next have the following formulas:

$$
(d \omega)^{0}=d\left(\omega^{0}\right), \quad(d \omega)^{\mathrm{I}}=d(\omega)^{\mathrm{I}}, \quad(d \omega)^{\mathrm{II}}=d\left(\omega^{\mathrm{II}}\right)
$$

for $\omega \in \mathscr{I}_{1}^{0}(M)$. In fact, taking account of (3.4), (3. 8) and (3.9), we have

$$
\begin{aligned}
2\left(d \omega^{0}\right)\left(X^{\mathrm{II}}, Y^{\mathrm{II}}\right) & =X^{\mathrm{II}} \omega^{0}\left(Y^{\mathrm{II}}\right)-Y^{\mathrm{II}} \omega^{0}\left(X^{\mathrm{II}}\right)-\omega^{0}\left(\left[X^{\mathrm{II}}, Y^{\mathrm{II}}\right]\right) \\
& =(X \omega(Y)-Y \omega(X)-\omega([X, Y]))^{0} \\
& =2((d \omega)(X, Y))^{0}=2(d \omega)^{0}\left(X^{\mathrm{II}}, Y^{\mathrm{II}}\right) .
\end{aligned}
$$


Therefore, according to the Remark stated in $\S 3$, we have $(d \omega)^{0}=d\left(\omega^{0}\right)$. By similar devices, we have the other formulas given in (4.6).

If we consider a differential form $\omega$ which has the local expression $\omega=f d x^{\imath_{1}} \wedge$ $\cdots \wedge d x^{\imath s}, f \in \mathscr{I}_{0}^{0}(U)$ in $\left(U,\left(x^{h}\right)\right)$, we obtain

$$
d \omega=d f \wedge d x^{\imath_{1}} \wedge \cdots \wedge d x^{\imath s}
$$

and hence, taking account of (4.3), (4.5) and (4.6),

$$
\begin{aligned}
(d \omega)^{\mathrm{I}} & =(d f)^{\mathrm{I}} \wedge\left(d x^{\imath_{1}} \wedge \cdots \wedge d x^{\imath s}\right)^{0}+(d f)^{0} \wedge\left(d x^{\imath_{1}} \wedge \cdots \wedge d x^{\imath s}\right)^{\mathrm{I}} \\
& =\left(d f^{\mathrm{I}}\right) \wedge\left(d x^{\imath_{1}} \wedge \cdots \wedge d x^{\imath s}\right)^{0}+\left(d f^{0}\right) \wedge\left(d x^{\imath_{1}} \wedge \cdots \wedge d x^{\imath s}\right)^{\mathrm{I}} \\
& =d\left(f^{\mathrm{I}}\left(d x^{\imath_{1}} \wedge \cdots \wedge d x^{\imath s}\right)^{0}+f^{0}\left(d x^{\imath_{1}} \wedge \cdots \wedge d x^{\imath s}\right)^{\mathrm{I}}\right) \\
& =d\left(f d x^{\imath_{1}} \wedge \cdots \wedge d x^{\imath s}\right)^{\mathrm{I}}=d\left(\omega^{\mathrm{I}}\right)
\end{aligned}
$$

by virtue of (3.10). Therefore, taking account of the identity $(\omega+\pi)^{\mathrm{I}}=\omega^{\mathrm{I}}+\pi^{\mathrm{I}}$ for $\omega, \pi \in \Lambda_{*}(M)$, we have $(d \omega)^{\mathrm{I}}=d\left(\omega^{\mathrm{I}}\right)$ for any element $\omega$ of $\Lambda_{*}(M)$. Similarly, we obtain $(d \omega)^{0}=d\left(\omega^{0}\right)$ and $(d \omega)^{\mathrm{II}}=d\left(\omega^{\mathrm{II}}\right)$ for any element $\omega$ of $\Lambda_{*}(M)$. Thus we have

Proposition 4.1. The formulas

$$
(d \omega)^{0}=d\left(\omega^{0}\right), \quad(d \omega)^{\mathrm{I}}=d\left(\omega^{\mathrm{I}}\right), \quad(d \omega)^{\mathrm{I}}=d\left(\omega^{\mathrm{II}}\right)
$$

hold for any element $\omega$ of $\Lambda_{*}(M)$.

Lie derivatives with respect to lifts. Denoting by $\mathcal{L}_{X}$ the operator of Lie derivation with respect to a vector field $X$, we have directly from (3.4)

$$
\begin{array}{lll}
\mathcal{L}_{X^{0}} f^{0}=0, & \mathcal{L}_{X^{0}} f^{\mathrm{I}}=0, & \mathcal{L}_{X^{0}} f^{\mathrm{II}}=\left(\mathcal{L}_{X} f\right)^{0}, \\
\mathcal{L}_{X^{\mathrm{I}}} f^{0}=0, & \mathcal{L}_{X^{\mathrm{I}}} f^{\mathrm{I}}=\frac{1}{2}\left(\mathcal{L}_{X} f\right)^{0}, & \mathcal{L}_{X^{\mathrm{I}}} f^{\mathrm{II}}=\left(\mathcal{L}_{X} f\right)^{\mathrm{I}}, \\
\mathcal{L}_{X \mathrm{II}} f^{0}=\left(\mathcal{L}_{X} f\right)^{0}, & \mathcal{L}_{X^{\mathrm{II}}} f^{\mathrm{I}}=\left(\mathcal{L}_{X} f\right)^{\mathrm{I}}, & \mathcal{L}_{X^{\mathrm{II}}} f^{\mathrm{II}}=\left(\mathcal{L}_{X} f\right)^{\mathrm{II}}
\end{array}
$$

for $f \in \mathscr{I}_{0}^{0}(M), X \in \mathscr{I}_{0}^{1}(M)$ and directly from (3.9)

$$
\mathcal{L}_{X^{0}} Y^{0}=0, \quad \mathcal{L}_{X^{0}} Y^{\mathrm{I}}=0, \quad \mathcal{L}_{X^{0}} Y^{\mathrm{II}}=\left(\mathcal{L}_{X} Y\right)^{0},
$$

$$
\begin{array}{lll}
\mathcal{L}_{X^{\mathrm{I}}} Y^{0}=0, & \mathcal{L}_{X^{\mathrm{I}}} Y^{\mathrm{I}}=\frac{1}{2}\left(\mathcal{L}_{X} Y\right)^{0}, & \mathcal{L}_{X^{\mathrm{I}}} Y^{\mathrm{II}}=\left(\mathcal{L}_{X} Y\right)^{\mathrm{I},} \\
\mathcal{L}_{X^{\mathrm{II}}} Y^{0}=\left(\mathcal{L}_{X} Y\right)^{0}, & \mathcal{L}_{X^{\mathrm{II}}} Y^{\mathrm{I}}=\left(\mathcal{L}_{X} Y\right)^{\mathrm{I},} & \mathcal{L}_{X^{\mathrm{II}}} Y^{\mathrm{II}}=\left(\mathcal{L}_{X} Y\right)^{\mathrm{II}}
\end{array}
$$

for $X, Y \in \mathscr{I}_{0}^{1}(M)$. We have now the following formulas: 


$$
\begin{aligned}
& \mathcal{L}_{X^{0}} \omega^{0}=0, \quad \mathcal{L}_{X^{0}} \omega^{\mathrm{I}}=0, \quad \mathcal{L}_{X^{0}} \omega^{\mathrm{II}}=\left(\mathcal{L}_{X} \omega\right)^{0}, \\
& \text { (4. 9) } \quad \mathcal{L}_{X}^{\mathrm{I}} \omega^{0}=0, \quad \mathcal{L}_{X}^{\mathrm{I}} \omega^{\mathrm{I}}=\frac{1}{2}\left(\mathcal{L}_{X} \omega\right)^{0}, \quad \mathcal{L}_{X}^{\mathrm{I}} \omega^{\mathrm{II}}=\left(\mathcal{L}_{X} \omega\right)^{\mathrm{I}}, \\
& \mathcal{L}_{X} \mathrm{II} \omega^{0}=\left(\mathcal{L}_{X} \omega\right)^{0}, \quad \mathcal{L}_{X} \mathrm{II} \omega^{\mathrm{I}}=\left(\mathcal{L}_{X} \omega\right)^{\mathrm{I}}, \quad \mathcal{L}_{X} \mathrm{II} \omega^{\mathrm{II}}=\left(\mathcal{L}_{X} \omega\right)^{\mathrm{II}}
\end{aligned}
$$

for $X \in \mathscr{I}_{0}^{1}(M), \omega \in \mathscr{I}_{1}^{0}(M)$. In fact, taking an arbitrary vector field $Y$ in $M$, we have

$$
\begin{aligned}
\left(\mathcal{L}_{X^{0}} \omega^{0}\right)\left(Y^{\mathrm{II}}\right) & =\mathcal{L}_{X^{0}}\left(\omega^{0}\left(Y^{\mathrm{II}}\right)\right)-\omega^{0}\left(\mathcal{L}_{X^{0}} Y^{\mathrm{II}}\right)=0 \\
\left(\mathcal{L}_{X} \mathrm{I} \omega^{\mathrm{I}}\right)\left(Y^{\mathrm{II}}\right) & =\mathcal{L}_{X^{\mathrm{I}}}\left(\omega^{\mathrm{I}}\left(Y^{\mathrm{II}}\right)\right)-\omega^{\mathrm{I}}\left(\mathcal{L}_{X^{\mathrm{I}}} Y^{\mathrm{II}}\right)=\frac{1}{2}\left(\mathcal{L}_{X}(\omega(Y))-\omega\left(\mathcal{L}_{X} Y\right)\right)^{0} \\
& =\frac{1}{2}\left(\left(\mathcal{L}_{X} \omega\right)(Y)\right)^{0}=\frac{1}{2}\left(\mathcal{L}_{X} \omega^{0}\left(Y^{\mathrm{II}}\right)\right. \\
\left(\mathcal{L}_{X} \mathrm{II} \omega^{\mathrm{II}}\right)\left(Y^{\mathrm{II}}\right) & =\mathcal{L}_{X} \mathrm{II}\left(\omega^{\mathrm{II}}\left(Y^{\mathrm{II}}\right)\right)-\omega^{\mathrm{II}}\left(\mathcal{L}_{X}{ }^{\mathrm{II}} Y^{\mathrm{II}}\right)=\left(\mathcal{L}_{X}(\omega(Y))-\omega\left(\mathcal{L}_{X} Y\right)\right)^{\mathrm{II}} \\
& =\left(\left(\mathcal{L}_{X} \omega\right)(Y)\right)^{\mathrm{II}}=\left(\mathcal{L}_{X} \omega\right)^{\mathrm{II}}\left(Y^{\mathrm{II}}\right)
\end{aligned}
$$

by virtue of (3.4), (3. 8), (4.7) and (4.8). Consequently, $Y$ being arbitrary, we find $\mathcal{L}_{X^{0}} \omega^{0}=0, \mathcal{L}_{X^{\mathrm{I}}} \omega^{\mathrm{I}}=(1 / 2)\left(\mathcal{L}_{X} \omega\right)^{0}, \mathcal{L}_{X^{\mathrm{II}}} \omega^{\mathrm{II}}=\left(\mathcal{L}_{X} \omega\right)^{\mathrm{II}}$. Similarly, we obtain the other formulas given in (4.9). We have here

Proposition 4.2. For any element $K$ of $\mathscr{I}(M)$ the formulas

$$
\begin{array}{lll}
\mathcal{L}_{X^{0}} K^{0}=0, & \mathcal{L}_{X^{0}} K^{\mathrm{I}}=0 & \mathcal{L}_{X^{0}} K^{\mathrm{II}}=\left(\mathcal{L}_{X} K\right)^{0}, \\
\mathcal{L}_{X^{\mathrm{I}}} K^{0}=0, & \mathcal{L}_{X^{\mathrm{I}}} K^{\mathrm{I}}=\frac{1}{2}\left(\mathcal{L}_{X} K\right)^{0}, & \mathcal{L}_{X^{\mathrm{I}}} K^{\mathrm{II}}=\left(\mathcal{L}_{X} K\right)^{\mathrm{I}}, \\
\mathcal{L}_{X^{\mathrm{II}}} K^{0}=\left(\mathcal{L}_{X} K\right)^{0}, & \mathcal{L}_{X^{\mathrm{II}}} K^{\mathrm{I}}=\left(\mathcal{L}_{X} K\right)^{\mathrm{I}}, & \mathcal{L}_{X^{\mathrm{II}}} K^{\mathrm{II}}=\left(\mathcal{L}_{X} K\right)^{\mathrm{II}}
\end{array}
$$

hold, $X$ being an arbitrary element of $\mathscr{I}_{0}^{1}(M)$.

Proof. These formulas have been already proved in (4.7), (4.8) and (4.9) respectively for $K$ belonging to $\mathscr{I}_{0}^{0}(M), \mathscr{I}_{0}^{1}(M)$ or $\mathscr{I}_{1}^{0}(M)$. Then we assume that these formulas are established for $K$ belonging to $\mathscr{I}_{s}^{r}(M)$, where $r \leqq p, s \leqq q$. Taking an arbitrary element $S$ of $\mathscr{I}_{m}^{l}(M)$ and an element $T$ of $\mathscr{I}_{q-m}^{p-l}(M)$, we have

$$
\begin{aligned}
\mathcal{L}_{X 0}(S \otimes T)^{0} & =\mathcal{L}_{X^{0}}\left(S^{0} \otimes T^{0}\right)=\left(\mathcal{L}_{X^{0}} S^{0}\right) \otimes T^{0}+S^{0} \otimes\left(\mathcal{L}_{X^{0}} T^{0}\right)=0 \\
\mathcal{L}_{X^{\mathrm{I}}}(S \otimes T)^{\mathrm{I}} & =\mathcal{L}_{X^{\mathrm{I}}}\left(S^{\mathrm{I}} \otimes T^{0}+S^{0} \otimes T^{\mathrm{I}}\right) \\
& =\left(\mathcal{L}_{X^{\mathrm{I}}} S^{\mathrm{I}}\right) \otimes T^{0}+S^{\mathrm{I}} \otimes\left(\mathcal{L}_{X^{\mathrm{I}}} T^{0}\right)+\left(\mathcal{L}_{X^{\mathrm{I}}} S^{0}\right) \otimes T^{\mathrm{I}}+S^{0} \otimes\left(\mathcal{L}_{X^{\mathrm{I}}} T^{\mathrm{I}}\right) \\
& =\frac{1}{2}\left(\left(\mathcal{L}_{X} S\right)^{0} \otimes T^{0}+S^{0} \otimes\left(\mathcal{L}_{X} T\right)^{0}\right) \\
& =\frac{1}{2}\left(\left(\mathcal{L}_{X} S\right) \otimes T+S \otimes\left(\mathcal{L}_{X} T\right)\right)^{0}=\frac{1}{2}\left(\mathcal{L}_{X} S \otimes T\right)^{0}
\end{aligned}
$$




$$
\begin{aligned}
& \mathcal{L}_{X^{\mathrm{II}}}(\mathrm{S} \otimes T)^{\mathrm{II}}=\mathcal{L}_{X^{\mathrm{II}}}\left(\mathrm{S}^{\mathrm{II}} \otimes T^{0}+2 \mathrm{~S}^{\mathrm{I}} \otimes T^{\mathrm{I}}+S^{0} \otimes T^{\mathrm{II}}\right) \\
& =\left(\mathcal{L}_{X} \mathrm{II} S^{\mathrm{II}}\right) \otimes T^{0}+S^{\mathrm{II}} \otimes\left(\mathcal{L}_{X} \mathrm{II} T^{0}\right)+2\left(\mathcal{L}_{X} \mathrm{II} S^{\mathrm{I}}\right) \otimes T^{\mathrm{I}} \\
& +2 S^{\mathrm{I}} \otimes\left(\mathcal{L}_{X} \mathrm{II} T^{\mathrm{I}}\right)+\left(\mathcal{L}_{X^{\mathrm{II}}} S^{0}\right) \otimes T^{\mathrm{II}}+S^{0} \otimes\left(\mathcal{L}_{X}{ }^{\mathrm{II}} S^{\mathrm{II}}\right) \\
& =\left(\left(\mathcal{L}_{X} S\right) \otimes T+S \otimes\left(\mathcal{L}_{X} T\right)\right)^{\mathrm{II}}=\left(\mathcal{L}_{X} S \otimes T\right)^{\mathrm{II}}
\end{aligned}
$$

by virtue of (4.1). Similarly, we can prove the other formulas given in Proposition 4. 2 for $K=S \otimes T$. Consequently, we have proved Proposition 4.2 as consequences of $\mathcal{L}_{X}(S+T)=\mathcal{L}_{X} S+\mathcal{L}_{X} T$ for $S, T \in \mathscr{I}_{s}^{r}(M)$.

Linear mappings $\alpha$ and $\beta$. We shall define a linear mapping $\alpha$ : $\mathscr{I}_{s}^{r}(M)$ $\rightarrow \mathscr{I}_{s-1}^{r}\left(T_{2}(M)\right)(s \geqq 1)$. Let $T$ be an element of $\mathscr{I}_{s}^{r}(M)$. Then $T^{\text {II }}$ is a correspondence

$$
T^{\mathrm{II}}: \quad\left(\tilde{X}_{1}, \cdots, \tilde{X}_{s}\right) \rightarrow T^{\mathrm{II}}\left(\tilde{X}_{1}, \cdots, \tilde{X}_{s}\right) \in \mathscr{I}_{0}^{r}\left(T_{2}(M)\right),
$$

$\tilde{X}_{1}, \cdots, \tilde{X}_{s}$ being arbitrary elements of $\mathscr{I}_{0}^{1}\left(T_{2}(M)\right)$. If we consider a correspondence $\alpha T$ such that

$$
\alpha T: \quad\left(\tilde{X}_{2}, \cdots, \tilde{X}_{s}\right) \rightarrow T^{\mathrm{II}}\left(A, \tilde{X}_{2}, \cdots, \tilde{X}_{s}\right) \in \mathscr{I}_{0}^{r}\left(T_{2}(M)\right),
$$

$\tilde{X}_{2}, \cdots, \tilde{X}_{s}$ being arbitrary elements of $\mathscr{I}_{0}^{1}\left(T_{2}(M)\right)$ and $A$ the vector field defined by (2. 5). Then $\alpha T$ is an element of $\mathscr{I}_{s-1}^{r}\left(T_{2}(M)\right)$. Then the correspondence $\alpha: T \rightarrow \alpha T$ determines a linear mapping $\alpha: \mathscr{I}_{s}^{r}(M) \rightarrow \mathscr{I}_{s-1}^{r}\left(T_{2}(M)\right)$. Thus we have from (4.10)

$$
\begin{aligned}
& \alpha \omega=\omega^{\mathrm{II}}(A) \quad \text { for } \quad \omega \in \mathscr{I}_{1}^{0}(M) \text {, } \\
& \alpha d f=f^{\mathrm{I}} \quad \text { for } f \in \mathscr{I}_{0}^{0}(M) \text {. }
\end{aligned}
$$

When $T$ has the form $T=\omega \otimes S, \omega \in \mathscr{I}_{1}^{0}(M), S \in \mathscr{I}(M)$, taking account of (4.1), we find

$$
\alpha T=(\alpha(1)) S^{0} \quad(T=\omega \otimes S)
$$

because of the formulas

$$
\omega^{0}(A)=0, \quad \omega^{\mathrm{I}}(A)=0, \quad \omega^{\mathrm{II}}(A)=\alpha \omega
$$

for $\omega \in \mathscr{I}_{1}^{0}(M)$, which are direct consequences of (2.5) and (3. 5).

We shall next define a linear mapping $\beta$ : $\mathscr{I}_{s}^{r}(M) \rightarrow \mathscr{I}_{s-1}^{r}\left(T_{2}(M)\right)(s \geqq 1)$. Let $T$ be an element of $\mathscr{I}_{s}^{r}(M)$. If we consider a correspondence

$$
\beta T: \quad\left(\tilde{X}_{2}, \cdots, \tilde{X}_{s}\right) \rightarrow T^{\mathrm{II}}\left(B, \tilde{X}_{2}, \cdots, \tilde{X}_{s}\right) \in \mathscr{I}_{s-1}^{r}\left(T_{2}(M)\right),
$$

$\tilde{X}_{2}, \cdots, X_{s}$ being arbitrary elements of $\mathscr{I}_{0}^{1}\left(T_{2}(M)\right)$ and $B$ the vector field defined by (2.5), then $\beta T$ is an element of $\mathscr{I}_{s-1}^{r}\left(T_{2}(M)\right)$. Thus the correspondence $\beta: T \rightarrow \beta T$ defines a linear mapping $\beta: \mathscr{I}_{s}^{r}(M) \rightarrow \mathscr{I}_{s-1}^{r}\left(T_{2}(M)\right)$. We have now 


$$
\begin{array}{clrl}
\beta \omega & =\omega^{\mathrm{II}}(B) & \text { for } & \omega \in \mathscr{I}_{1}^{0}(M), \\
\beta(d f) & =f^{\mathrm{II}} & \text { for } & f \in \mathscr{I}_{0}^{0}(M) .
\end{array}
$$

When $T$ has the form $T=\omega \otimes S, \omega \in \mathscr{I}_{1}^{0}(M), S \in \mathscr{I}(M)$, taking account of (4. 1), we obtain

$$
\beta T=(\beta \omega) S^{0}+(\alpha \omega) S^{I}
$$

by virtue of the formulas

$$
\omega^{0}(B)=0, \quad \omega^{\mathrm{I}}(B)=\frac{1}{2} \alpha \omega, \quad \omega^{\mathrm{T}}(B)=\beta \omega
$$

for $\omega \in \mathscr{I}_{1}^{0}(M)$, which are direct consequences of (2.5) and (3.5).

\section{§5. Local expressions.}

In this section, we would like to find local expressions of the lifts of tensor fields in $M$. By components of a tensor field $T$ in $M$ we always mean those of $T$ in coordinate neighborhood $\left(U,\left(x^{h}\right)\right)$ of $M$ and by components of a tensor field $\tilde{T}$ in $T_{2}(M)$ those of $\tilde{T}$ with respect to the induced coordinates $\left(\xi^{A}\right)=\left(x^{h}, y^{h}, z^{h}\right)$ in $\pi_{2}^{-1}(U)$. The local expression of a function $f$, a vector field $X$ and a 1-form $\omega$ have been already given by (2. 2), (3.1) and (3.5) respectively.

Tensor fields of type $(1,1)$. Let $F$ be an element of $\mathscr{I}_{1}^{1}(M)$, which is expressed by

$$
F=F_{i}^{h} d x^{2} \otimes \frac{\partial}{\partial x^{h}}
$$

in $\left.\left(U, x^{h}\right)\right)$. Taking the 0 -th lift, we find

$$
\begin{aligned}
F^{0} & =\left(F_{i}{ }^{h} d x^{2} \otimes \frac{\partial}{\partial x^{h}}\right)^{0} \\
& =\left(F_{i}{ }^{h}\right)^{0} d x^{2} \otimes \frac{\partial}{\partial y^{h}}
\end{aligned}
$$

by virtue of (3.10) and (4.1). Taking the 1st lift, we have

$$
\begin{aligned}
F^{\mathrm{I}} & =\left(F_{i}^{h} d x^{\imath} \otimes \frac{\partial}{\partial x^{h}}\right)^{\mathrm{I}} \\
& =\left(F_{i}{ }^{h}\right)^{0}\left(d y^{\imath} \otimes \frac{\partial}{\partial x^{h}}+\frac{1}{2} d x^{2} \otimes \frac{\partial}{\partial y^{h}}\right)+\left(F_{i}{ }^{h}\right)^{\mathrm{I}} d y^{\imath} \otimes \frac{\partial}{\partial z^{h}}
\end{aligned}
$$

by virtue of (3.10) and (4.1). Taking the 2nd lift, we obtain 


$$
\begin{aligned}
F^{\mathrm{II}}= & \left(F_{i}{ }^{h} d x^{2} \otimes \frac{\partial}{\partial x^{h}}\right)^{\mathrm{II}} \\
= & \left(F_{i}{ }^{h}\right)^{0}\left(d z^{2} \otimes \frac{\partial}{\partial y^{h}}+d y^{\imath} \otimes \frac{\partial}{\partial y^{h}}+d x^{2} \otimes \frac{\partial}{\partial x^{h}}\right) \\
& +\left(F_{i}{ }^{h}\right)^{\mathrm{I}}\left(2 d y^{2} \otimes \frac{\partial}{\partial z^{h}}+d x^{\imath} \otimes \frac{\partial}{\partial y^{h}}\right)+\left(F_{i}{ }^{h}\right)^{\mathrm{II}}\left(d x^{2} \otimes \frac{\partial}{\partial z^{h}}\right)
\end{aligned}
$$

by virtue of (3.10) and (4.1). Therefore we see that the lifts $F^{\mathbf{0}}, F^{\mathrm{I}}$ and $F^{\mathrm{II}}$ of $F$ have respectively the components of the form

$$
F^{0}:\left(\begin{array}{ccc}
0 & 0 & 0 \\
0 & 0 & 0 \\
F_{i}^{h} & 0 & 0
\end{array}\right), \quad F^{\mathbf{I}}:\left(\begin{array}{ccc}
0 & 0 & 0 \\
\frac{1}{2} F_{i}^{h} & 0 & 0 \\
y^{s} \partial_{s} F_{i}{ }^{h} & F_{i}^{h} & 0
\end{array}\right)
$$

$$
F^{\mathrm{II}}:\left(\begin{array}{ccc}
F_{i}{ }^{h} & 0 & 0 \\
y^{s} \partial_{s} F_{i}^{h} & F_{i}{ }^{h} & 0 \\
z^{s} \partial_{s} F_{i}^{h}+y^{t} y^{s} \partial_{t} \partial_{s} F_{i}^{h} & 2 y^{s} \partial_{s} F_{i}^{h} & F_{i}{ }^{h}
\end{array}\right) \text {, }
$$

where $F_{i}^{h}$ denote the components of $F$. We have from (5.1)

Proposition 5.1. A tensor field $F$ of type $(1,1)$ is of rank $r$, if and only if $F^{0}$ is of rank $r$, if and only if $F^{\mathrm{I}}$ is of rank $2 r$, or, if and only if $F^{\mathrm{II}}$ is of rank $3 r$.

Let $I$ be the identity tensor field of type $(1,1)$. Then, substituting $F_{i}^{h}=\delta_{i}^{h}$ in (5. 1), we find

$$
I^{0}:\left(\begin{array}{lll}
0 & 0 & 0 \\
0 & 0 & 0 \\
I & 0 & 0
\end{array}\right), \quad I^{\mathrm{I}}:\left(\begin{array}{ccc}
0 & 0 & 0 \\
\frac{1}{2} I & 0 & 0 \\
0 & I & 0
\end{array}\right), \quad I^{\mathrm{II}}:\left(\begin{array}{ccc}
I & 0 & 0 \\
0 & I & 0 \\
0 & 0 & I
\end{array}\right) .
$$

Therefore the 2nd lift $I^{\text {II }}$ of the identity tensor field I of type $(1,1)$ is the identity tensor field of type $(1,1)$ in $T_{2}(M)$. We have from (3.1) and (5.2)

$$
I^{0} X^{0}=0, \quad I^{0} X^{\mathrm{I}}=0, \quad I^{0} X^{\mathrm{II}}=X^{0},
$$

$$
I^{\mathrm{I}} X^{0}=0, \quad I^{\mathrm{I}} X^{\mathrm{I}}=\frac{1}{2} X^{0}, \quad I^{\mathrm{I}} X^{\mathrm{II}}=X^{\mathrm{II}}
$$

for $X \in \mathscr{I}_{0}^{1}(M)$.

Tensor fields of type $(0,2)$. Let $g$ be an element of $\mathscr{I}_{2}^{0}(M)$. Then we can easily verify that its lifts $g^{0}, g^{\mathrm{I}}$ and $g^{\mathrm{II}}$ have respectively components of the form 
(5. 4)

$$
g^{0}:\left(\begin{array}{ccc}
g_{j i} & 0 & 0 \\
0 & 0 & 0 \\
0 & 0 & 0
\end{array}\right), \quad g^{\mathrm{I}:}\left(\begin{array}{ccc}
y^{s} \partial_{s} g_{j i} & g_{j i} & 0 \\
g_{j i} & 0 & 0 \\
0 & 0 & 0
\end{array}\right) \text {, }
$$

$$
g^{\mathrm{II}}:\left(\begin{array}{ccc}
z^{s} \partial_{s} g_{j i}+y^{t} y^{s} \partial_{t} \partial_{s} g_{j i} & 2 y^{s} \partial_{s} g_{j i} & g_{j i} \\
2 y^{s} \partial_{s} g_{j i} & 2 y^{s} \partial_{s} g_{j i} & 0 \\
g_{j i} & 0 & 0
\end{array}\right)
$$

where $g_{j i}$ denote the components of $g$.

Given an element $\tilde{h}$ of $\mathscr{I}_{2}^{0}\left(T_{2}(M)\right)$, we denote by

$$
\tilde{h}(d \xi, d \xi)=\tilde{h}_{c B} d \xi^{c} d \xi^{B}
$$

the quadratic differential form corresponding to $\tilde{h}$, if $\tilde{h}$ is symmetric, $\tilde{h}_{C B}$ being the components of $\tilde{h}$. Let $g$ be a pseudo-Riemannian metric in $M$. Then, taking account of (5.4), we obtain

$$
\begin{aligned}
g^{0}(d \xi, d \xi) & =g_{j i} d x^{\jmath} d x^{\imath}, \\
g^{\mathrm{I}}(d \xi, d \xi) & =2 g_{j i} d x^{j} \delta y^{2}, \\
g^{\mathrm{II}}(d \xi, d \xi) & =2 g_{j i} d x^{j} \delta v^{\imath}+2 g_{j i} \delta y^{j} \delta y^{\imath},
\end{aligned}
$$

the differential forms $\delta y^{h}$ and $\delta z^{h}$ being defined respectively by

$$
\begin{aligned}
& \delta y^{h}=d y^{h}+\left\{\begin{array}{c}
h \\
s
\end{array}\right\} y^{s} d x^{2}
\end{aligned}
$$

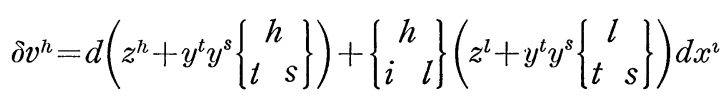

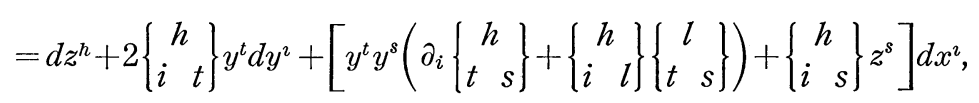

where $\left\{\begin{array}{c}h \\ j\end{array} i\right\}$ denote the Christoffel's symbols constructed from $g_{j i}$ and $v^{h}$ are defined by

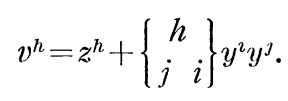

We have, from (5.5),

Proposition 5.2. Let $g$ be a pseudo-Riemannian metric in $M$ (with $r$ positive and $n-r$ negative signs). Then $g^{\mathrm{II}}$ is a pseudo-Riemannian metric in $T_{2}(M)$ (with $n+r$ negative and $2 n-r$ positive signs).

Let $\varphi$ be a 2-form of the maximum rank in $M$. Then $\varphi^{\mathrm{II}}$ is also a 2 -form of the maximum rank in $T_{2}(M)$ because of (5.4). When $\varphi=d \eta, \eta$ being a 1-form, 
then $\varphi^{\mathrm{II}}=d\left(\eta^{\mathrm{II}}\right)$ as an immediate consequence of Proposition 4. 1. Thus we have

Proposition 5.3. If $\varphi$ is a 2-form defining an (almost) symplectic structure in $M$, then $\varphi^{\mathrm{II}}$ defines an (almost) symplectic structure in $T_{2}(M)$.

Tensor fields of type $(2,0)$. Let $G$ be a tensor field of type $(2,0)$ in $M$. Then we can easily verify that its lifts $G^{0}, G^{\mathrm{I}}$ and $G^{\mathrm{I}}$ have respectively components of the form

$$
G^{0}:\left(\begin{array}{ccc}
0 & 0 & 0 \\
0 & 0 & 0 \\
0 & 0 & G^{j i}
\end{array}\right), \quad G^{\mathrm{I}}:\left(\begin{array}{ccc}
0 & 0 & 0 \\
0 & 0 & \frac{1}{2} G^{j i} \\
0 & \frac{1}{2} G^{j i} & y^{s} \partial_{s} G^{j i}
\end{array}\right),
$$

$$
G^{\mathrm{II}}:\left(\begin{array}{ccc}
0 & 0 & G^{j i} \\
0 & \frac{1}{2} G^{j i} & y^{s} \partial_{s} G^{j i} \\
G^{j i} & y^{s} \partial_{s} G^{j i} & z^{s} \partial_{s} G^{j i}+y^{t} y^{s} \partial_{t} \partial_{s} G^{j i}
\end{array}\right),
$$

where $G^{j i}$ denote the components of $G$.

Tensor fields $\alpha T$ and $\beta T$. We shall give the local expressions of $\alpha T$ and $\beta T$ defined in $\S 4$. Taking account of (2.5) and (3. 5), we have from (4.11) and (4.15)

$$
\alpha \omega=y^{i} \omega_{i}, \quad \beta \omega=z^{k} \partial_{k} \omega_{i}+y^{\jmath} y^{i} \partial_{j} \omega_{i} \quad \text { for } \quad \omega \in \mathscr{I}_{1}^{0}(M)
$$

with respect to the induced coordinates $\left(x^{h}, y^{h}, z^{h}\right)$ in $\pi_{2}^{-1}(U)$, where $\omega_{i}$ denote the components of $\omega$. Especially, we have from (5.8)

$$
\alpha\left(d x^{h}\right)=y^{h}, \quad \beta\left(d x^{h}\right)=z^{h}
$$

in $\pi_{2}^{-1}(U)$.

Let $T$ be an element of $\mathscr{I}_{s}^{r}(M)(s \geqq 1)$ and assume that $T$ has the expression

$$
T=T_{\imath_{1} \imath_{2} \cdots v_{s}}{ }^{h_{1} \cdots h_{r}} d x^{\imath_{1}} \otimes d x^{\imath_{2}} \otimes \cdots \otimes d x^{\imath_{s}} \otimes \frac{\partial}{\partial x^{h_{1}}} \otimes \cdots \otimes \frac{\partial}{\partial x^{h_{r}}}
$$

in $\left(U,\left(x^{h}\right)\right)$. Then, taking account of (4.12), we have

$$
\begin{aligned}
\alpha T & =\alpha\left(d x^{\jmath} \otimes\left(T_{j i_{2} \cdots \imath_{s}}{ }^{h_{1} \cdots h_{r}} d x^{\imath_{2}} \otimes \cdots \otimes d x^{\imath_{s}} \otimes \frac{\partial}{\partial x^{h_{\imath}}} \otimes \cdots \otimes \frac{\partial}{\partial x^{h r}}\right)\right) \\
& =\alpha\left(d x^{j}\right)\left(T_{j i_{2} \cdots \imath_{s}}{ }^{h_{1} \cdots h_{r}}\right)^{0} d x^{\imath_{2}} \otimes \cdots \otimes d x^{\imath_{s}} \otimes \frac{\partial}{\partial z^{h_{1}}} \otimes \cdots \otimes \frac{\partial}{\partial z^{h_{r}}}
\end{aligned}
$$

by virtue of (3. 10), since $\alpha(T+S)=\alpha T+\alpha S$ for $T, S \in I_{s}^{r}(M)$. Thus, according to (5. 9), we obtain

$$
\alpha T=\left(y^{j} T_{j i_{2} \cdots l_{s}}^{h_{1} \cdots h^{\prime}}\right) d x^{2_{2}} \otimes \cdots \otimes d x^{c_{s}} \otimes \frac{\partial}{\partial z^{h_{1}}} \otimes \cdots \otimes \frac{\partial}{\partial z^{h r}}
$$


with respect to the induced coordinates $\left(\xi^{A}\right)$ in $\pi_{2}^{-1}(U)$. Especially, for any element $F$ of $\mathscr{I}_{1}^{1}(M), \alpha T$ has components of the form

$$
\alpha F:\left(\begin{array}{c}
0 \\
0 \\
y^{i} F_{i}^{h}
\end{array}\right),
$$

$F_{i}^{h}$ denoting the components of $F$. For an element $S$ of $\mathscr{I}_{2}^{1}(M), \alpha S$ has components of the form

$$
\alpha S:\left(\begin{array}{ccc}
0 & 0 & 0 \\
0 & 0 & 0 \\
y^{j} S_{j i}{ }^{h} & 0 & 0
\end{array}\right)
$$

where $S_{j i}{ }^{h}$ denote the components of $S$.

Let $F$ be an element of $\mathscr{I}_{1}^{1}(M)$ with local expression

$$
F=F_{i}^{h} d x^{2} \otimes \frac{\partial}{\partial x^{h}}
$$

Then, taking account of (4.16), we have

$$
\begin{aligned}
\beta F & =\beta\left(d x^{i} \otimes\left(F_{i}^{h} \frac{\partial}{\partial x^{h}}\right)\right) \\
& =\beta\left(d x^{i}\right)\left(F_{i}^{h} \frac{\partial}{\partial x^{h}}\right)^{0}+\alpha\left(d x^{i}\right)\left(F_{i}^{h} \frac{\partial}{\partial x^{h}}\right)^{\mathrm{I}} \\
& =\beta\left(d x^{i}\right)\left(F_{i}^{h}\right)^{0} \frac{\partial}{\partial z^{h}}+\left(d x^{i}\right)\left(\left(F_{i}^{h}\right)^{\mathrm{I}} \frac{\partial}{\partial z^{h}}+\frac{1}{2}\left(F_{i}^{h}\right)^{0} \frac{\partial}{\partial y^{h}}\right) \\
& =\frac{1}{2} y^{i}\left(F_{i}^{h}\right)^{0} \frac{\partial}{\partial y^{h}}+\left(z^{2}\left(F_{i}^{h}\right)^{0}+y^{2}\left(F_{i}^{h}\right)^{\mathrm{I}}\right) \frac{\partial}{\partial z^{h}}
\end{aligned}
$$

by virtue of (3.10) and (5. 9), since $\beta(T+S)=\beta T+\beta S$ for $T, S \in \mathscr{I}_{s}^{r}(M)$. This means that $\beta F$ has components of the form

$$
\beta F:\left(\begin{array}{c}
0 \\
\frac{1}{2} y^{i} F_{i}{ }^{h} \\
z^{i} F_{i}^{h}+y^{\jmath} y^{i} \partial_{j} F_{i}{ }^{h}
\end{array}\right)
$$

for any element $F \in \mathscr{I}_{1}^{1}(M)$, where $F_{i}^{h}$ denote the components of $F$.

By similar devices, we see that, for an element $S$ of $\mathscr{I}_{2}^{1}(M), \beta S$ has components of the form 


$$
\beta S:\left(\begin{array}{ccc}
0 & 0 & 0 \\
\frac{1}{2} y^{k} S_{k i}{ }^{h} & 0 & 0 \\
z^{k} S_{k i}{ }^{h}+y^{t} y^{s} \partial_{t} S_{s i}{ }^{h} & y^{k} S_{k i}{ }^{h} & 0
\end{array}\right) \text {, }
$$

where $S_{j i}{ }^{h}$ denote the components of $S$.

If we take account of (3.1) and (5.8), we obtain the following formulas:

$$
\begin{aligned}
X^{0}(\alpha \omega) & =0, & X^{0}(\beta \omega) & =(\omega(X))^{0}, \\
X^{\mathrm{I}}(\alpha \omega) & =\frac{1}{2}(\omega(X))^{0}, & X^{\mathrm{I}}(\beta \omega) & =\alpha\left(\mathcal{L}_{X} \omega\right)+(\alpha(d \omega))(X), \\
X^{\mathrm{II}}(\alpha \omega) & =\alpha\left(\mathcal{L}_{X} \omega\right), & X^{\mathrm{II}}(\beta \omega) & =\beta\left(\mathcal{L}_{X} \omega\right)
\end{aligned}
$$

for $\omega \in \mathscr{I}_{1}^{0}(M)$ and $X \in \mathscr{I}_{0}^{1}(M)$.

\section{$\S 6$. Lifts of tensor fields of type $(1,1)$.}

Formulas. Let $F$ be an element of $\mathscr{I}_{1}^{1}(M)$. Then, taking account of (3.1) and (5.1), we find easily the following

$$
\begin{array}{lll}
F^{0} X^{0}=0, & F^{0} X^{\mathrm{I}}=0 & F^{0} X^{\mathrm{II}}=(F X)^{0}, \\
F^{\mathrm{I}} X^{0}=0, & F^{\mathrm{I}} X^{\mathrm{I}}=\frac{1}{2}(F X)^{0}, & F^{\mathrm{I}} X^{\mathrm{II}}=(F X)^{\mathrm{I}}, \\
F^{\mathrm{II}} X^{0}=(F X)^{0}, & F^{\mathrm{II}} X^{\mathrm{I}}=(F X)^{\mathrm{I}}, & F^{\mathrm{II}} X^{\mathrm{II}}=(F X)^{\mathrm{II}}
\end{array}
$$

for $F \in \mathscr{I}_{1}^{1}(M), X$ being an arbitrary element of $\mathscr{I}_{0}^{1}(M)$.

For any two elements $F$ and $G$ of $\mathscr{I}_{1}^{1}(M)$, we defined an element $F G$ of $\mathscr{I}_{1}^{1}(M)$ by $(F G) X=F(G X), X$ being an arbitrary element of $\mathscr{I}_{0}^{1}(M)$. Then we find the following formulas:

$$
\begin{array}{rlrl}
G^{0} F^{0} & =0, & G^{0} F^{\mathrm{I}}=0 & G^{0} F^{\mathrm{II}}=(G F)^{0}, \\
G^{\mathrm{I}} F^{0}=0, & G^{\mathrm{I}} F^{\mathrm{I}}=\frac{1}{2}(G F)^{0}, & G^{\mathrm{I}} F^{\mathrm{II}}=(G F)^{\mathrm{I}}, \\
G^{\mathrm{II}} F^{0}=(G F)^{0}, & G^{\mathrm{II}} F^{\mathrm{I}}=(G F)^{\mathrm{I}}, & G^{\mathrm{II}} F^{\mathrm{II}}=(G F)^{\mathrm{II}}
\end{array}
$$

for $G, F \in \mathscr{I}_{2}^{1}(M)$. In fact, taking account of (6.1), we have

$$
\begin{aligned}
\left(G^{0} F^{0}\right) X^{\mathrm{II}} & =G^{0}\left(F^{0} X^{\mathrm{II}}\right)=0 \\
\left(G^{\mathrm{I}} F^{\mathrm{I}}\right) X^{\mathrm{II}} & =G^{\mathrm{I}}\left(F^{\mathrm{I}} X^{\mathrm{II}}\right)=G^{\mathrm{I}}(F X)^{\mathrm{I}}=\frac{1}{2}(G(F X))^{\mathrm{I}}=\frac{1}{2}(G F)^{0} X^{\mathrm{II}} \\
\left(G^{\mathrm{II}} F^{\mathrm{II}}\right) X^{\mathrm{II}} & =G^{\mathrm{II}}\left(F^{\mathrm{II}} X^{\mathrm{II}}\right)=G^{\mathrm{II}}(F X)^{\mathrm{II}}=(G(F X))^{\mathrm{II}}=(G F)^{\mathrm{II}} X^{\mathrm{II}}
\end{aligned}
$$

for any element $X$ of $\mathscr{I}_{0}^{1}(M)$. Thus we have $G^{0} F^{0}=(G F)^{0}, G^{\mathrm{I}} F^{\mathrm{I}}=(1 / 2)(G F)^{0}$ and 
$G^{\mathrm{II}} F^{\mathrm{II}}=(G F)^{\mathrm{II}}$. The other formulas given in (6.2) are proved in a similar way.

We see from (6.2) that, for any element $F$ of $\mathscr{I}_{1}^{1}(M), F^{0}, F^{\mathrm{I}}$ and $F^{\text {II }}$ are commutative with each other and the identities

$$
\left(F^{0}\right)^{2}=0, \quad\left(F^{\mathrm{I}}\right)^{3}=0 \quad \text { for } \quad F \in \mathscr{I}_{1}^{1}(M)
$$

hold.

Let $P(t)$ be a polynomial of $t$ and $F \in \mathscr{I}_{1}^{1}(M)$. Then, taking account of (6.2), we obtain

$$
(P(F))^{\mathrm{II}}=P\left(F^{\mathrm{II}}\right)
$$

and hence, for example,

$$
\left(F^{2}+I\right)^{\mathrm{II}}=\left(F^{\mathrm{II}}\right)^{2}+I, \quad\left(F^{3}+F\right)^{\mathrm{II}}=\left(F^{\mathrm{II}}\right)^{3}+F^{\mathrm{II}}
$$

for any element $F$ of $\mathscr{I}_{1}^{1}(M)$.

A tensor field $F$ of type $(1,1)$ is called an almost complex structure if $F^{2}+I=0$. A tensor field $F$ is called an $f$-structure of rank $r$ if $F^{3}+F=0$ and $F$ is of rank $r$ everywhere. Thus, taking account of Proposition 5.1, we have from (6.5)

Proposition 6.1. Let $F$ be an element of $\mathscr{I}_{1}^{1}(M)$. Then $F^{\mathrm{II}}$ is an almost complex structure in $T_{2}(M)$ if and only if $F$ is so in $M$. $F^{\mathrm{II}}$ is an $f$-structure of rank $3 r$ in $T_{2}(M)$ if and only if $F$ is an f-structure of rank $r$ in $M$.

Contraction in Lifts. Let $F$ be an element of $\mathscr{I}_{1}^{1}(M)$. We denote by $c(F)$ the element of $\mathscr{I}_{0}^{0}(M)$ obtained by contraction, i.e., $c(F)=F_{i}{ }^{\imath}$ if $F$ has components $F_{i}^{h}$. Then we have from $(5.1)$

$$
c\left(F^{0}\right)=0, \quad c\left(F^{\mathrm{I}}\right)=0, \quad c\left(F^{\mathrm{II}}\right)=3(c(F))^{0}
$$

for $F \in \mathscr{I}_{1}^{1}(M)$. For example, we have

$$
c\left((\omega \otimes X)^{0}\right)=0, \quad c\left((\omega \otimes X)^{\mathrm{I}}\right)=0, \quad c\left((\omega \otimes X)^{\mathrm{II}}\right)=3(\omega(X))^{0},
$$

$X$ and $\omega$ being respectively elements of $\mathscr{I}_{0}^{1}(M)$ and $\mathscr{I}_{1}^{0}(M)$.

Torsion tensors and Nijenhuis tensors. Let $S$ be an element of $\mathscr{I}_{2}^{1}(M)$ such that $S=Z \otimes \omega \otimes \pi, Z \in \mathscr{I}_{0}^{1}(M), \omega, \pi \in \mathscr{I}_{1}^{0}(M)$. Then, taking account of (3. 8) and (4.1), we have the following formulas:

$$
\begin{gathered}
S^{0}\left(X^{\mathrm{II}}, Y^{\mathrm{II}}\right)=(S(X, Y))^{0}, \quad S^{\mathrm{I}}\left(X^{\mathrm{II}}, Y^{\mathrm{II}}\right)=(S(X, Y))^{\mathrm{I}}, \\
S^{\mathrm{II}}\left(X^{\mathrm{II}}, Y^{\mathrm{II}}\right)=(S(X, Y))^{\mathrm{II}}
\end{gathered}
$$

for $S \in \mathscr{I}_{2}^{1}(M), X$ and $Y$ being arbitrary elements of $\mathscr{I}_{0}^{1}(M)$.

Let there be given two elements $G$ and $F$ of $\mathscr{I}_{1}^{1}(M)$. Then their torsion tensor $N_{F, G}$ is by definition a tensor field of type $(1,2)$ given by

$$
\begin{aligned}
N_{F, G}(X, Y)= & {[F X, G Y]+[G X, F Y]+F G[X, Y]+G F[X, Y] } \\
& -F[X, G Y]-F[G X, Y]-G[X, F Y]-G[F X, Y],
\end{aligned}
$$


$X$ and $Y$ being arbitrary elements of $\mathscr{I}_{0}^{1}(M)$. Thus, taking account of (3.9), (6.1) and (6.2), we obtain

$$
\begin{aligned}
N_{F^{0}, G^{0}}\left(X^{\mathrm{II}}, Y^{\mathrm{II}}\right) & =0, \quad N_{F^{0}, G^{\mathrm{I}}}\left(X^{\mathrm{II}}, Y^{\mathrm{II}}\right)=0, \\
F_{F^{0}, G^{\mathrm{II}}}\left(X^{\mathrm{II}}, Y^{\mathrm{II}}\right) & =\left(N_{F, G}(X, Y)\right)^{0}, \\
N_{F^{\mathrm{I}}, G^{\mathrm{I}}}\left(X^{\mathrm{II}}, Y^{\mathrm{II}}\right) & =\frac{1}{2}\left(N_{F, G}(X, Y)\right)^{0}, \\
N_{F^{\mathrm{I}}, G^{\mathrm{II}}}\left(X^{\mathrm{II}}, Y^{\mathrm{II}}\right) & =\left(N_{F, G}(X, Y)\right)^{\mathrm{I}}, \\
N_{F^{\mathrm{II}}, G^{\mathrm{II}}}\left(X^{\mathrm{II}}, Y^{\mathrm{II}}\right) & =\left(N_{F, G}(X, Y)\right)^{\mathrm{II}},
\end{aligned}
$$

$X$ and $Y$ being arbitrary elements of $\mathscr{I}_{0}^{1}(M)$. Thus, we have from (6.10)

$$
N_{F^{0}, G^{0}}=0, \quad N_{F^{\mathrm{I}}, G^{\mathrm{I}}}=\frac{1}{2}\left(N_{F, G}\right)^{0},
$$

$$
\begin{array}{ll}
N_{F^{0}, G \mathrm{I}}=0, & N_{F^{\mathrm{I}}, G \mathrm{II}}=\left(N_{F, G}\right)^{\mathrm{I}}, \\
N_{F^{0}, G \mathrm{II}}=\left(N_{F, G}\right)^{0}, & N_{F^{\mathrm{II}}, G \mathrm{II}}=\left(N_{F, G}\right)^{\mathrm{II}}
\end{array}
$$

for $F, G \in \mathscr{I}_{1}^{1}(M)$ by virtue of (6.8).

The Nijenhuis tensor $N_{F}$ of an element $F$ of $\mathscr{I}_{1}^{1}(M)$ is defined by $N_{F}=(1 / 2) N_{F, F}$. Thus we have from (6.11)

PROPOSITION 6.2. For any element $F$ of $\mathscr{I}_{1}^{1}(M)$

$$
N_{F^{0} 0}=0, \quad N_{F^{\prime} \mathrm{I}}=\frac{1}{2}\left(N_{F^{\prime}}\right)^{0}, \quad N_{F^{\prime} \mathrm{I}}=\left(N_{F^{\prime}}\right)^{\mathrm{II}}
$$

hold.

Proposition 6. 3. Let $F$ be an almost complex structure in $M$. Then the almost complex structure $F^{\mathrm{II}}$ is a complex structure in $T_{2}(M)$ if and only if $F$ is so in $M$.

\section{$\S 7$. Lifts of affine connections.}

Lifts of affine connections. Let $\nabla$ be an affine connection in $M$, which has coefficients $\Gamma_{j}{ }^{h} \imath$ in $\left(U,\left(x^{h}\right)\right)$. We now introduce in $\pi_{2}{ }^{-1}(U)$ an affine connection $\nabla^{\text {II }}$ having coefficients $\tilde{\Gamma}_{C^{A} B}{ }_{B}$ with respect to the induced coordinates $\left(\xi^{A}\right)=\left(x^{h}, y^{h}, z^{h}\right)$ such that

$$
\left(\tilde{\Gamma}_{C}{ }^{h}{ }_{B}\right)=\left(\begin{array}{ccc}
\left(\Gamma_{j}{ }^{h}\right)^{0} & 0 & 0 \\
0 & 0 & 0 \\
0 & 0 & 0
\end{array}\right)
$$

for each fixed index $h$, 


$$
\left(\tilde{\Gamma}_{C}{ }^{h_{B}}{ }_{B}\right)=\left(\begin{array}{ccc}
\left(\Gamma_{j}{ }^{h} \imath\right)^{\mathrm{I}} & \left(\Gamma_{\jmath}{ }^{h} \imath\right)^{0} & 0 \\
\left(\Gamma_{\jmath}{ }^{h}\right)^{0} & 0 & 0 \\
0 & 0 & 0
\end{array}\right)
$$

for each fixed $\bar{h}$ and

$$
\left(\tilde{\Gamma}_{C^{\bar{h}}}{ }_{B}\right)=\left(\begin{array}{ccc}
\left(\Gamma_{j}{ }^{h} \imath\right)^{\mathrm{II}} & 2\left(\Gamma_{j}{ }^{h} \imath\right)^{\mathrm{I}} & \left(\Gamma_{j}{ }^{h} i\right)^{0} \\
2\left(\Gamma_{j}{ }^{h} \imath\right)^{\mathrm{I}} & 2\left(\Gamma_{j}{ }^{h} i\right)^{0} & 0 \\
\left(\Gamma_{j}{ }^{h} \imath\right)^{0} & 0 & 0
\end{array}\right)
$$

for each fixed index $\overline{\bar{h}}$, where $\left(\Gamma_{j}{ }^{h} i\right)^{0},\left(\Gamma_{j}{ }^{h}\right)^{\mathrm{I}}$ and $\left(\Gamma_{j}{ }^{h}\right)^{\mathrm{II}}$ denote respectively the 0 -th, the 1st and the 2nd lifts of the functions $\Gamma_{j}{ }^{h} \imath$ given in $\left(U,\left(x^{h}\right)\right)$. We note here that the transformation law of coefficients $\Gamma_{j}^{h}{ }_{\imath}$ of an affine connection is given by

$$
\Gamma_{j^{\prime}}{ }^{h^{\prime}}{ }^{\prime}{ }^{\prime}=\frac{\partial x^{h^{\prime}}}{\partial x^{h}}\left(\frac{\partial x^{j}}{\partial x^{j^{\prime}}} \frac{\partial x^{\imath}}{\partial x^{i^{\prime}}} \Gamma_{j^{\prime}}{ }^{h}+\frac{\partial^{2} x^{h}}{\partial x^{j^{\prime}} \partial x^{i^{\prime}}}\right)
$$

in $U \cap U^{\prime}$. Thus, taking account of (1.5), (1.6), (7.1), (7.2) and (7.3), we know by virtue of (7.4) that the affine connection $V^{\text {II }}$ introduced above in each $\pi_{2}^{-1}(U)$ determines globally in $T_{2}(M)$ an affine connection, which is denoted also by $\nabla^{\text {III }}$. The affine connection $\nabla^{\text {II }}$ constructed thus in $T_{2}(M)$ is called the lift of the affine connection $\nabla$ given in $M$.

We obtain here the following formulas:

$$
\begin{array}{lll}
\nabla_{X^{0}}^{\mathrm{II}} Y^{0}=0, & \nabla_{X^{0}}^{\mathrm{II}} Y^{\mathrm{I}}=0, & \nabla_{X^{0}}^{\mathrm{II}} Y^{\mathrm{II}}=\left(\nabla_{X} Y\right)^{0}, \\
\nabla_{X^{\mathrm{I}}}^{\mathrm{II}} Y^{0}=0, & \nabla_{X^{\mathrm{I}}}^{\mathrm{II}} Y^{\mathrm{I}}=\frac{1}{2}\left(\nabla_{X} Y\right)^{0}, & \nabla_{X^{\mathrm{I}}}^{\mathrm{II}} Y^{\mathrm{II}}=\left(\nabla_{X} Y\right)^{\mathrm{I},} \\
\nabla_{X^{\mathrm{II}}}^{\mathrm{II}} Y^{0}=\left(\nabla_{X} Y\right)^{0}, & \nabla_{X^{\mathrm{II}}}^{\mathrm{II}} Y^{\mathrm{I}}=\left(\nabla_{X} Y\right)^{\mathrm{I}} & \nabla_{X^{\mathrm{II}}}^{\mathrm{II}} Y^{\mathrm{II}}=\left(\nabla_{X} Y\right)^{\mathrm{II}}
\end{array}
$$

for $X, Y \in \mathscr{I}_{0}^{1}(M)$. In fact, taking account of (3.1), (7.1), (7.2) and (7.3), we see that $\nabla_{X^{I I}}^{\mathrm{II}} Y^{\mathrm{II}}$ has components of the form

$$
\begin{aligned}
& \left(\nabla_{X^{\mathrm{II}}}^{\mathrm{II}} Y^{\mathrm{II}}\right)^{h}=X^{j}\left(\frac{\partial Y^{h}}{\partial x^{j}}+\Gamma_{j^{\prime}}{ }_{\imath} Y^{i}\right)=X^{j} \nabla_{\jmath} Y^{h}, \\
& \left(\nabla_{X^{\mathrm{II}}}^{\mathrm{II}} Y^{\mathrm{II}}\right)^{\bar{h}}=X^{j}\left[\frac{\partial}{\partial x^{j}}\left(y^{s} \partial_{s} Y^{h}\right)+\left(y^{s} \partial_{s} \Gamma_{j}{ }_{\imath}\right) Y^{i}+\Gamma_{j}{ }_{i}{ }_{i}\left(y^{s} \partial_{s} Y^{i}\right)\right] \\
& +\left(y^{s} \partial_{s} X^{j}\right)\left[\frac{\partial}{\partial y^{j}}\left(y^{s} \partial_{s} Y^{h}\right)+I^{1}{ }_{j}{ }_{\imath} Y^{i}\right] \\
& =y^{s} \partial_{s}\left(X^{j}\left(\frac{\partial Y^{h}}{\partial x^{j}}+\Gamma_{j}{ }_{\imath} Y^{i}\right)\right)=y^{s} \partial_{s}\left(X^{j} \nabla_{J} Y^{h}\right)_{s}
\end{aligned}
$$




$$
\begin{aligned}
\left(\nabla_{X^{\mathrm{II}}}^{\mathrm{II}} Y^{\mathrm{II}}\right)^{\overline{\bar{h}}}= & X^{\jmath}\left[\frac{\partial}{\partial x^{j}}\left(z^{s} \partial_{s} Y^{h}+y^{t} y^{s} \partial_{t} \partial_{s} Y^{h}\right)+\left(z^{s} \partial_{s} \Gamma_{j}{ }_{i}+y^{t} y^{s} \partial_{t} \partial_{s} I_{j}{ }^{h}{ }_{\imath}\right) Y^{\imath}\right. \\
& \left.+2\left(y^{s} \partial_{s} \Gamma_{j}{ }^{h} i\right)\left(y^{t} \partial_{t} Y^{i}\right)+\Gamma_{j}{ }^{h}{ }_{i}\left(z^{s} \partial_{s} Y^{i}+y^{t} y^{s} \partial_{t} \partial_{s} Y^{i}\right)\right] \\
& +\left(y^{k} \partial_{k} X^{j}\right)\left[\frac{\partial}{\partial y^{\jmath}}\left(z^{s} \partial_{s} Y^{h}+y^{t} y^{s} \partial_{t} \partial_{s} Y^{h}\right)+2\left(y^{s} \partial_{s} \Gamma_{j}{ }^{h}{ }_{i}\right) Y^{i}+2 \Gamma_{j}{ }_{j}{ }_{i}\left(y^{s} \partial_{s} Y^{i}\right)\right] \\
& +\left(z^{k} \partial_{k} X^{\jmath}+y^{m} y^{l} \partial_{m} \partial_{l} X^{j}\right)\left[\frac{\partial}{\partial z^{\jmath}}\left(z^{s} \partial_{s} Y^{h}+y^{t} y^{s} \partial_{t} \partial_{s} Y^{h}\right)+\Gamma_{j}{ }_{j}{ }_{\imath} Y^{i}\right] \\
= & z^{s} \partial_{s}\left(X^{j}\left(\partial_{\jmath} Y^{h}+\Gamma_{j}{ }_{\imath} Y^{i}\right)\right)+y^{t} y^{s} \partial_{t} \partial_{s}\left(X^{j}\left(\partial_{\jmath} Y^{h}+\Gamma_{j}{ }_{\imath}{ }_{\imath} Y^{i}\right)\right) \\
= & z^{s} \partial_{s}\left(X^{j} \nabla_{\jmath} Y^{h}\right)+y^{t} y^{s} \partial_{t} \partial_{s}\left(X^{j} \nabla_{\jmath} Y^{h}\right) .
\end{aligned}
$$

Therefore we find $\nabla_{X}^{\mathrm{II}} Y^{\mathrm{II}}=\left(\nabla_{X} Y\right)^{\mathrm{II}}$. Similarly, we obtain the other formulas given in (7.5).

Comparing (7.5) with (4.2) or (6.1), we find easily the formulas

$$
\nabla^{\mathrm{II}} Y^{\mathrm{O}}=(\nabla Y)^{0}, \quad \nabla^{\mathrm{II}} Y^{\mathrm{I}}=(\nabla Y)^{\mathrm{I}}, \quad \nabla^{\mathrm{II}} Y^{\mathrm{II}}=(\nabla Y)^{\mathrm{II}}
$$

for $Y \in \mathscr{I}_{0}^{1}(M)$.

We also obtain the following formulas:

$$
\begin{array}{lll}
\nabla_{X^{0}}^{\mathrm{II}} \omega^{0}=0, & \nabla_{X^{0}}^{\mathrm{II}} \omega^{\mathrm{I}}=0, & \nabla_{X^{0}}^{\mathrm{II}} \omega^{\mathrm{II}}=\left(\nabla_{X} \omega\right)^{0}, \\
\nabla_{X^{\mathrm{I}}}^{\mathrm{II}} \omega^{0}=0, & \nabla_{X^{\mathrm{I}}}^{\mathrm{II}} \omega^{\mathrm{I}}=\frac{1}{2}\left(\nabla_{X} \omega\right)^{0}, & \nabla_{X^{\mathrm{I}}}^{\mathrm{II}} \omega^{\mathrm{II}}=\left(\nabla_{X} \omega\right)^{\mathrm{I}} \\
\nabla_{X^{\mathrm{II}}}^{\mathrm{II}} \omega^{0}=\left(\nabla_{X} \omega\right)^{0}, & \nabla_{X^{\mathrm{II}}}^{\mathrm{II}} \omega^{\mathrm{I}}=\left(\nabla_{X} \omega\right)^{\mathrm{I}}, & \nabla_{X^{\mathrm{II}}}^{\mathrm{II}} \omega^{\mathrm{II}}=\left(\nabla_{X} \omega\right)^{\mathrm{II}}
\end{array}
$$

for $X \in \mathscr{I}_{0}^{1}(M), \omega \in \mathscr{I}_{1}^{0}(M)$. In fact, taking an arbitrary element $Y$ of $\mathscr{I}_{0}^{1}(M)$, we have

$$
\begin{aligned}
\left(\nabla_{X \mathrm{II}}^{\mathrm{II}} \omega^{\mathrm{II}}\right)\left(Y^{\mathrm{II}}\right) & =\nabla_{X}^{\mathrm{II}}\left(\omega^{\mathrm{II}}\left(Y^{\mathrm{II}}\right)\right)-\omega^{\mathrm{II}}\left(\nabla_{X}^{\mathrm{II}} Y^{\mathrm{II}}\right) \\
& =\left(\nabla_{X}(\omega(Y))-\omega\left(\nabla_{X} Y\right)\right)^{\mathrm{II}} \\
& =\left(\left(\nabla_{X} \omega\right)(Y)\right)^{\mathrm{II}}=\left(\nabla_{X} \omega\right)^{\mathrm{II}}\left(Y^{\mathrm{II}}\right)
\end{aligned}
$$

by virtue of (3. 8) and (7.5). Thus we have $\nabla_{X^{I I}}^{\mathrm{II}} \omega^{\mathrm{II}}=\left(\nabla_{X} \omega\right)^{\mathrm{II}}$, because $Y$ is arbitrary. The other formulas given in (7.7) are proved in a similar way.

We have from (7.7) the formulas

$$
\nabla^{\mathrm{II}} \omega^{0}=(\nabla \omega)^{0}, \quad \nabla^{\mathrm{II}} \omega^{\mathrm{I}}=(\nabla \omega)^{\mathrm{I}}, \quad \nabla^{\mathrm{II}} \omega^{\mathrm{II}}=(\nabla \omega)^{\mathrm{II}}
$$

for $\omega \in \mathscr{I}_{1}^{0}(M)$. In fact, we have from (4.2) and (7.7)

$$
\gamma_{X} \mathrm{II}\left(\nabla^{\mathrm{II}} \omega^{\mathrm{II}}\right)=\nabla_{X}^{\mathrm{II}} \omega^{\mathrm{II}}=\left(\nabla_{X} \omega\right)^{\mathrm{II}}=\left(\gamma_{X}(\nabla \omega)\right)^{\mathrm{II}}=\gamma_{X} \mathrm{II}(\nabla \omega)^{\mathrm{II}}
$$

for any element $X$ of $\mathscr{L}_{0}^{1}(M)$. Thus we have $\nabla^{\mathrm{II}} \omega^{\mathrm{II}}=(\nabla \omega)^{\mathrm{II}}$. Similarly, we can prove the other formulas given in (7.8). 
We have here from (7.6) and (7.8)

Proposition 7.1. For any element $K$ of $\mathscr{I}(M)$

$$
\nabla^{\mathrm{II}} K^{0}=(\nabla K)^{0}, \quad \nabla^{\mathrm{II}} K^{\mathrm{I}}=(\nabla K)^{\mathrm{I}}, \quad \nabla^{\mathrm{II}} K^{\mathrm{II}}=(\nabla K)^{\mathrm{II}}
$$

hold.

We have directly from Proposition 7.1 the formulas

$$
\begin{array}{lll}
\nabla_{X}^{\mathrm{II}} K^{0}=0, & \nabla_{X}^{\mathrm{II}} K^{\mathrm{I}}=0, & \nabla_{X}^{\mathrm{II}} K^{\mathrm{II}}=\left(\nabla_{X} K\right)^{0}, \\
\nabla_{X^{\mathrm{I}}}^{\mathrm{II}} K^{0}=0, & \nabla_{X^{\mathrm{I}}}^{\mathrm{II}} K^{\mathrm{I}}=\frac{1}{2}\left(\nabla_{X} K\right)^{0}, & \nabla_{X^{\mathrm{I}}}^{\mathrm{II}} K^{\mathrm{II}}=\left(\nabla_{X} K\right)^{\mathrm{I},} \\
\nabla_{X^{\mathrm{II}}}^{\mathrm{II}} K^{0}=\left(\nabla_{X} K\right)^{0}, & \nabla_{X^{\mathrm{II}}}^{\mathrm{II}} K^{\mathrm{I}}=\left(\nabla_{X} K\right)^{\mathrm{I},} & \nabla_{X^{\mathrm{II}}}^{\mathrm{II}} K^{\mathrm{II}}=\left(\nabla_{X} K\right)^{\mathrm{II}}
\end{array}
$$

for $X \in \mathscr{I}_{0}^{1}(M), K \in \mathscr{I}_{s}^{r}(M)$ by virtue of (4.2).

The Curvature and the torsion tensors. Denoting by $T$ the torsion tensor of an affine connection $\nabla$ in $M$, we have by definition

$$
T(X, Y)=\left(\nabla_{X} Y-\nabla_{Y} X\right)-[X, Y] \quad \text { for } \quad X, Y \in \mathscr{I}_{0}^{1}(M) \text {. }
$$

Taking the second lift, we obtain

$$
\begin{aligned}
(T(X, Y))^{\mathrm{II}} & =\left(\nabla_{X^{\mathrm{II}}}^{\mathrm{II}} Y^{\mathrm{II}}-\nabla_{Y^{\mathrm{II}}}^{\mathrm{II}} X^{\mathrm{II}}\right)-\left[X^{\mathrm{II}}, Y^{\mathrm{II}}\right] \\
& =\widetilde{T}\left(X^{\mathrm{II}}, Y^{\mathrm{II}}\right)
\end{aligned}
$$

by virtue of Proposition 7.1 and (3.9), where $\widetilde{T}$ denotes the torsion tensor of $\nabla^{11}$. This equation implies, together with (6.8), $T^{\mathrm{II}}\left(X^{\mathrm{II}}, Y^{\mathrm{II}}\right)=\widetilde{T}\left(X^{\mathrm{II}}, Y^{\mathrm{II}}\right)$. Thus, we have $T^{\mathrm{II}}=\widetilde{T}$, since $X$ and $Y$ are arbitrary. Therefore we have

Proposition 7.2. The torsion tensor of the lift $\nabla^{\text {II }}$ of an affine connection $\nabla$ given in $M$ coincides with the 2nd lift $T^{\mathrm{II}}$ of the torsion tensor $T$ of $\nabla$.

The curvature tensor $R$ of an affine connection $\nabla$ in $M$ is a tensor field of type $(1,3)$ such that, for any two elements $X$ and $Y$ of $\mathscr{I}_{0}^{1}(M), R(X, Y)$ is an element of $\mathscr{I}_{1}^{1}(M)$ satisfying the condition

$$
R(X, Y) Z=\left(\nabla_{X} \nabla_{Y} Z-\nabla_{Y} \nabla_{X} Z\right)-\nabla_{[X, Y]} Z
$$

for any element $Z$ of $\mathscr{I}_{1}^{1}(M)$. Taking the 2 nd lift, we find

$$
\begin{aligned}
(R(X, Y) Z)^{\mathrm{II}} & =\left(\nabla_{X^{\mathrm{II}}}^{\mathrm{II}} \nabla_{Y^{\mathrm{II}}}^{\mathrm{II}} Z^{\mathrm{II}}-\nabla_{Y^{\mathrm{II}}}^{\mathrm{II}} V_{X^{\mathrm{II}}}^{\mathrm{II}} Z^{\mathrm{II}}\right)-\nabla_{\left[X^{\mathrm{II}}, Y^{\mathrm{II}}\right.}^{\mathrm{II}} Z^{\mathrm{II}} \\
& =\widetilde{R}\left(X^{\mathrm{II}}, Y^{\mathrm{II}}\right) Z^{\mathrm{II}}
\end{aligned}
$$

by virtue of Proposition 7.1 and (3.9), where $\tilde{R}$ denotes the curvature tensor of $\nabla^{\text {II }}$. On the other hand, we can verify $(R(X, Y) Z)^{\mathrm{II}}=R^{\mathrm{II}}\left(X^{\mathrm{II}}, Y^{\mathrm{II}}\right) Z^{\text {II }}$ by virtue of $\nabla_{X}{ }^{\mathrm{II}} K^{\mathrm{II}}=\left(V_{X} K\right)^{\mathrm{II}}$ given in (4.2). Therefore we find $\tilde{R}\left(X^{\mathrm{II}}, Y^{\mathrm{II}}\right) Z^{\mathrm{II}}=R^{\mathrm{II}}\left(X^{\mathrm{II}}, Y^{\mathrm{II}}\right) Z^{\mathrm{II}}$, which implies $\widetilde{R}=R^{11}$ since $X, Y$ and $Z$ are arbitrarily taken. Thus we have 
Proposition 7.3. The curvature tensor of the lift $\nabla^{\mathrm{II}}$ of an affine connection $\nabla$ given in $M$ coincides with the 2 nd lift $R^{\mathrm{II}}$ of the curvature tensor $R$ of $\nabla$.

As a corollary to Propositions 7.1 and 7.3, we have

Proposition 7.4. Let $T$ and $R$ be respectively the torsion and the curvature tensors of an affine connection $\nabla$ given in $M$. According as $T=0, \nabla T=0, R=0$ or $\nabla R=0$, we have $T^{\mathrm{II}}=0, \nabla^{\mathrm{II}} T^{\mathrm{II}}=0, R^{\mathrm{II}}=0$ or $\nabla^{\mathrm{II}} R^{\mathrm{II}}=0$. In particular, $T_{2}(M)$ is locally symmetric with respect to the lift $\nabla^{\mathrm{II}}$ of $\nabla$ if and only if $M$ is so with respect to $\nabla$.

Let $g$ be a pseudo-Riemannian metric in $M$ and $\nabla$ the Riemannian connection determined by $g$. Then we have from Proposition 7.1

$$
\nabla^{\mathrm{II}} g^{\mathrm{II}}=(\nabla g)^{\mathrm{II}}=0 .
$$

On the other hand, since $\nabla$ is torsionless, so is $\nabla^{11}$ by virtue of Proposition 7.2. Consequently, $\nabla^{\text {II }}$ should coincide with the Riemannian connection determined by $g^{\text {II }}$. Thus we have

Proposition 7. 5. Let g be a pseudo-Riemannian metric in $M$ and $\nabla$ its Riemannian connection. Then the lift $\nabla^{\mathrm{II}}$ of $\nabla$ is the Riemannian connection determined by the 2 nd lift $g^{\mathrm{II}}$ of $g$.

We have from Propositions 7.4 and 7.5

Propositions 7.6. Let $g$ be a pseudo-Riemannian metric in $M$. Then $T_{2}(M)$ is locally symmetric with respect to $g^{\mathrm{II}}$ if and only if $M$ is so with respect to $g$.

Let $P$ be an element of $\mathscr{I}_{3}^{1}(M)$. Then we have from (4.2)

$$
\gamma_{X^{\mathrm{II}} \gamma_{Y} \mathrm{II}} P^{\mathrm{II}}=\left(\gamma_{X} \gamma_{Y} P\right)^{\mathrm{II}},
$$

in which both sides belong to $\mathscr{I}_{1}^{1}\left(T_{2}(M)\right)$.

If we take account of (6.6), we find

$$
c\left(\gamma_{X^{\mathrm{II}} \gamma_{Y} \mathrm{II}} P^{\mathrm{II}}\right)=c\left(\left(\gamma_{X} \gamma_{Y} P\right)^{\mathrm{II}}\right)=3\left(c\left(\gamma_{X} \gamma_{Y} P\right)\right)^{0},
$$

which implies

Proposition 7.7. Let $g$ be a pseudo-Riemannian metric in $M$. Then the Ricci tensor $\tilde{K}$ of $g^{\mathrm{II}}$ coincides with $3 K^{0}$, where $K$ denotes the Ricci tensor of $g$.

If $g^{\mathrm{II}}$ is an Einstein metric in $T_{2}(M)$, we have $\tilde{K}=a g^{\mathrm{II}}$ with a constant $a, \tilde{K}$ being the Ricci tensor of $g^{\text {II }}$. However, we have from proposition $7.7 \tilde{K}=3 K^{0}$. Thus we have $a g^{I I}=3 K^{0}$, which, together with (5.4), implies $a=0$. Therefore we have

Proposition 7. 8. Let $g$ be a pseudo-Riemannian metric in $M$. If $g^{\mathrm{II}}$ is an 
Einstein metric in $T_{2}(M)$, then $g^{\mathrm{II}}$ is of zero Ricci tensor. If $g^{\mathrm{II}}$ is of constant curvature, then $g^{\mathrm{II}}$ is locally flat.

Let $\widetilde{K}_{C B}$ denote the components of the Ricci tensor $\tilde{K}=3 K^{0}$ of $g^{\text {II }}$ and $\tilde{G}^{C B}$ the contravariant components of $g^{\mathrm{II}}$. Then, taking account of (5.4) and (5.7), we have $\tilde{k}=\tilde{K}_{C B} \tilde{G}^{C B}=3\left(K_{j i} g^{j i}\right)^{0}$, where $K_{j i}$ denote the components of the Ricci tensor of $g$ and $g^{j i}$ the contravariant components of $g$. Thus we have

Proposition 7.9. Let $g$ be a pseudo-Riemannian metric in $M$. Let $k$ and $\tilde{k}$ be the curvature scalars of $g$ and $g^{\mathrm{II}}$ respectively. Then $\tilde{k}=3 k^{0}$. If $g$ is of constant curvature scalar, so is $g^{\mathrm{II}}$.

A pseudo-Riemannian metric $g$ is of constant curvature $k$ in $M$ if

$$
R(X, Y) Z=k(g(Z, Y) X-g(Z, X) Y) \quad \text { for } \quad X, Y \in \mathscr{I}_{0}^{1}(M)
$$

with a constant $k, R$ denoting the curvature tensor of $g$. Taking the 2nd lift, we have

$$
\begin{aligned}
R^{\mathrm{II}}\left(X^{\mathrm{II}}, Y^{\mathrm{II}}\right) Z^{\mathrm{II}=} & (R(X, Y) Z)^{\mathrm{II}}=(k(g(Z, Y) X-g(Z, X) Y))^{\mathrm{II}} \\
= & k\left[g^{\mathrm{II}}\left(Z^{\mathrm{II}}, Y^{\mathrm{II}}\right) X^{0}+2 g^{\mathrm{I}}\left(Z^{\mathrm{II}}, Y^{\mathrm{II}}\right) X^{\mathrm{I}}+g^{0}\left(Z^{\mathrm{II}}, Y^{\mathrm{II}}\right) X^{\mathrm{II}}\right. \\
& \left.-g^{\mathrm{II}}\left(Z^{\mathrm{II}}, X^{\mathrm{II}}\right) Y^{0}-2 g^{\mathrm{I}}\left(Z^{\mathrm{II}}, X^{\mathrm{II}}\right) Y^{\mathrm{I}}-g^{0}\left(Z^{\mathrm{II}}, X^{\mathrm{II}}\right) Y^{\mathrm{II}}\right] .
\end{aligned}
$$

If we take account of $I^{0} X^{\mathrm{II}}=X^{0}, I^{\mathrm{I}} X^{\mathrm{II}}=X^{\mathrm{I}}$ given in (5.3), we have from the equation above

$$
\begin{aligned}
& R^{\mathrm{II}}\left(X^{\mathrm{II}}, Y^{\mathrm{II}}\right) Z^{\mathrm{II}} \\
= & k\left[g^{\mathrm{II}}\left(Z^{\mathrm{II}}, Y^{\mathrm{II}}\right) I^{0} X^{\mathrm{II}}+2 g^{\mathrm{I}}\left(Z^{\mathrm{II}}, Y^{\mathrm{II}}\right) I^{\mathrm{I}} X^{\mathrm{II}}+g^{0}\left(Z^{\mathrm{II}}, Y^{\mathrm{II}}\right) X^{\mathrm{II}}\right. \\
& \left.-g^{\mathrm{II}}\left(Z^{\mathrm{II}}, X^{\mathrm{II}}\right) I^{0} Y^{\mathrm{II}}-2 g^{\mathrm{I}}\left(Z^{\mathrm{II}}, X^{\mathrm{II}}\right) I^{\mathrm{I}} Y^{\mathrm{II}}-g^{0}\left(Z^{\mathrm{II}}, X^{\mathrm{II}}\right) Y^{\mathrm{II}}\right]
\end{aligned}
$$

which gives the curvature tensor $R^{\mathrm{II}}$ of $g^{\mathrm{II}}$ in $T_{2}(M)$ when $g$ is of constant curvature in $M$.

\section{§ 8. Lifts of infinitesimal transformations.}

Let $g$ be a pseudo-Riemannian metric in $M$. Then we have from Proposition 4. 2

$$
\mathcal{L}_{X^{0}} g^{\mathrm{II}}=\left(\mathcal{L}_{X} g\right)^{0}, \quad \mathcal{L}_{X^{\mathrm{I}}} g^{\mathrm{II}}=\left(\mathcal{L}_{X} g\right)^{\mathrm{I}}, \quad \mathcal{L}_{X^{\mathrm{II}}} g^{\mathrm{II}}=\left(\mathcal{L}_{X} g\right)^{\mathrm{II}}
$$

for any element $X$ of $\mathscr{I}_{0}^{1}(M)$. If $X$ is a Killing vector field with respect to $g$, i.e., if $\mathcal{L}_{X} g=0$, then $X^{0}, X^{\mathrm{I}}$ and $X^{\mathrm{II}}$ are so with respect to $g^{\mathrm{II}}$. Thus, taking account of (5.2), we have

Proposition 8.1. Let $g$ be a pseudo-Riemannian metric in $M$. If $X$ is a 
Killing vector field with respect to $g$ in $M$, then $X^{0}, X^{\mathrm{I}}, X^{\mathrm{II}}$ are all Killing vector fields with respect to the pseudo-Riemannian metric $g^{\mathrm{II}}$ in $T_{2}(M)$.

Similarly, taking account of Proposition 6.1, we have

PRoposition 8.2. If $X$ is an (almost) analytic vector field in $M$ with respect to an (almost) complex structure $F$, i.e., if $\mathcal{L}_{X} F=0$, then $X^{0}, X^{\mathrm{I}}$ and $X^{\mathrm{II}}$ are so also in $T_{2}(M)$ with respect to the (almost) complex structure $F^{\mathrm{II}}$.

Let $X$ be a conformal Killing vector field in $M$ with respect to a pseudoRiemannian metric $g$. Then we have $\mathcal{L}_{X} g=a g, a \in \mathscr{I}_{0}^{0}(M)$. Thus, taking account of (8. 1), we obtain

$$
\mathcal{L}_{X}{ }^{\mathrm{II}} g^{\mathrm{II}}=a^{\mathrm{II}} g^{0}+2 a^{\mathrm{I}} g^{\mathrm{I}}+a^{0} g^{\mathrm{II}},
$$

which implies

Proposition 8. 3. Let $X$ be a conformal Killing vector field in $M$ with respect to a pseudo-Riemannian metric g. Then $X^{\mathrm{II}}$ is conformal in $T_{2}(M)$ with respect to $g^{\mathrm{II}}$ if and only if $X$ is homothetic, i.e., if and only if $\mathcal{L}_{X} g=a g$ holds with a constant $a$. If this is the case, $X^{\mathrm{II}}$ is necessarily homothetic.

Let $\nabla$ be an affine connection in $M$. Then, for any element $X$ of $\mathscr{I}_{0}^{1}(M)$, the Lie derivative of $\nabla$ with respect to $X$ is an element $\mathcal{L}_{X} \nabla$ of $\mathscr{I}_{2}^{1}(M)$ defined by

$$
\left(\mathcal{L}_{X} \nabla\right)(Y, Z)=\mathcal{L}_{X}\left(\nabla_{Y} Z\right)-\mathcal{L}_{Y}\left(\nabla_{X} Z\right)-\mathcal{L}_{[X, Y]} Z,
$$

$X, Y$ and $Z$ belonging to $\mathscr{I}_{0}^{1}(M)$. Thus, taking account of (3.9), (8.2) and Proposition 4.2 , we obtain

$$
\begin{aligned}
\left(\mathcal{L}_{X^{\mathrm{II}}} \nabla^{\mathrm{II}}\right)\left(Y^{\mathrm{II}}, Z^{\mathrm{II}}\right) & =\mathcal{L}_{X^{\mathrm{II}}}\left(\nabla_{Y^{\mathrm{II}}}^{\mathrm{II}} Z^{\mathrm{II}}\right)-\nabla_{X^{\mathrm{II}}}^{\mathrm{II}}\left(\mathcal{L}_{X^{\mathrm{II}}} Z^{\mathrm{II}}\right)-\nabla_{\left[X^{\mathrm{II}}, \boldsymbol{Y}^{\mathrm{II}}\right.}^{\mathrm{II}} Z^{\mathrm{II}} \\
& =\left(\mathcal{L}_{X}\left(\nabla_{Y} Z\right)-\nabla_{Y}\left(\mathcal{L}_{X} Z\right)-\nabla_{[X, Y]} Z\right)^{\mathrm{II}} \\
& =\left(\left(\mathcal{L}_{X} \nabla\right)(Y, Z)\right)^{\mathrm{II}}=\left(\mathcal{L}_{X} \nabla\right)^{\mathrm{II}}\left(Y^{\mathrm{II}}, Z^{\mathrm{II}}\right)
\end{aligned}
$$

for any element $Y$ and $Z$ of $\mathscr{I}_{0}^{1}(M)$. Thus we find

$$
\mathcal{L}_{X^{\mathrm{II}}} \nabla^{\mathrm{II}}=\left(\mathcal{L}_{X} \nabla\right)^{\mathrm{II}} .
$$

Similarly, we can prove the other formulas given in Proposition 8.4. Thus we have

Proposition 8. 4. Let $\nabla$ be an affine connection in $M$. Then, for any element $X$ of $\mathscr{I}_{0}^{1}(M)$, the formulas

$$
\mathcal{L}_{X^{0}} \nabla^{\mathrm{II}}=\left(\mathcal{L}_{X} \nabla\right)^{0}, \quad \mathcal{L}_{X^{\mathrm{I}}} \nabla^{\mathrm{II}}=\left(\mathcal{L}_{X} \nabla\right)^{\mathrm{I}}, \quad \mathcal{L}_{X^{\mathrm{II}}} \nabla^{\mathrm{II}}=\left(\mathcal{L}_{X} \nabla\right)^{\mathrm{II}}
$$

hold in $T_{2}(M)$. 
A vector field $X$ is called an infinitesimal affine transformation with respect to an affine connection $\nabla$ if $\mathcal{L}_{X} \nabla=0$. As a consequence of Proposition 8.4, we have

Proposition 8.5. Let $\nabla$ be an affine connection in $M$. If $X$ is an infinitesimal affine transformation in $M$ with respect to $\nabla$, then $X^{0}, X^{\mathrm{I}}$ and $X^{\mathrm{II}}$ are so also in $T_{2}(M)$ with respect to $\nabla^{\mathrm{II}}$.

A vector field $X$ in $M$ is called an infinitesimal projective transformation with respect to an affine connection $\nabla$ if

$$
\left(\mathcal{L}_{X} \nabla\right)(Y, Z)=\eta(Z) Y+\eta(Y) Z
$$

$\eta$ being a certain element of $\mathscr{I}_{1}^{0}(M)$. Taking the 2 nd lift, we have

$$
\begin{aligned}
\left(\mathcal{L}_{X^{\mathrm{II}}} \nabla^{\mathrm{II}}\right)\left(Y^{\mathrm{II}}, Z^{\mathrm{II}}\right)= & \eta^{\mathrm{II}}\left(Z^{\mathrm{II}}\right) Y^{0}+2 \eta^{\mathrm{I}}\left(Z^{\mathrm{II}}\right) Y^{\mathrm{I}}+\eta^{0}\left(Z^{\mathrm{II}}\right) Y^{\mathrm{II}} \\
& +\eta^{\mathrm{II}}\left(Y^{\mathrm{II}}\right) Z^{0}+2 \eta^{\mathrm{I}}\left(Y^{\mathrm{II}}\right) Z^{\mathrm{I}}+\eta^{0}\left(Y^{\mathrm{II}}\right) Z^{\mathrm{II}}
\end{aligned}
$$

by virtue of Propositton 8.4. Thus we have

Proposition 8.6. Let $X$ be an infinitesimal projective transformation in $M$ with respect to an affine connection $\nabla$. Then $X^{I I}$ is an infinitesimal projective transformation with respect to $\nabla^{\mathrm{II}}$ if and onlf if $X$ is affine. If this is the case, $X^{\mathrm{II}}$ is necessarily affine with respect to $\nabla^{\mathrm{II}}$.

Let $X$ be an element of $\mathscr{I}_{0}^{1}(M)$ and $\exp (t X)$ denote a local 1-parameter group of transformations of $M$ generated by $X$. Then, according to (1.10) and (3.1), $X^{\text {II }}$ generates a local 1-parameter group of $T_{2}(M)$ and

$$
\exp \left(t X^{\mathrm{II}}\right)=(\exp (t X)) *
$$

holds. Hence we have

Proposition 8.7. If a vector field $X$ in $M$ is complete in the sense that it generates a global 1-parameter group of transformations of $M$, then $X^{\mathrm{II}}$ is also complete in $T_{2}(M)$.

REMARK. From the local expressions (3.1) of $X^{0}$ and $X^{\mathrm{I}}$, we see immediately that $X^{0}$ and $X^{\mathrm{I}}$ are complete in $T_{2}(M)$ whether $X$ is complete in $M$ or not.

Taking account of the Remark stated above, we have, from Propositions 8. 1 and 8.7,

Proposition 8.8. If $M$ is homogeneous pseudo-Riemannian manifold with 
metric $g$, so is $T_{2}(M)$ with metric $g^{\mathrm{II}}$.

Similarly, we have from Proposition 8. 2

PROPOSITION 8.9. If $M$ is homogeneous (almost) complex manifold with (almost) complex structure $F$, so is $T_{2}(M)$ with (almost) complex structure $F^{\mathrm{II}}$.

Similarly, we have from Proposition 8. 5

PROPOSITION 8.10. If a group $G$ of affine transformations of $M$ with respect to an affine connection $\nabla$ is transitive in $M$, the group $G^{*}$ of affine transformations of $T_{2}(M)$ with respect to $\nabla^{\mathrm{II}}$ is transitive in $T_{2}(M)$, where $G^{*}$ denotes the group of transformations generated by vector fields $X^{0}, X^{\mathrm{I}}$ and $X^{\mathrm{II}}, X$ in $M$ being an arbitrary element belonging to the Lie algebra of vector fields generating $G$.

Let $M$ be a pseudo-Riemannian (resp. affine) symmetric space with metric $g$ (resp. connection $\nabla$ ). If we take an arbitrary point $\mathrm{P}$ in $M$, then there exists in $M$ a symmetry $S_{\mathrm{P}}$ with center $\mathrm{P}$, that is to say, $S_{\mathrm{P}}$ is in $M$ an isometry of $g$ (resp. an affine transformation of $V)$ such that $S_{\mathrm{P}}(\mathrm{P})=\mathrm{P},\left(S_{\mathrm{P}}\right)^{2}=$ identity. We note here that $M$ is identified with the zero-cross section $\bar{M}$ of $T_{2}(M)$, which is defined by equations $y^{h}=0, z^{h}=0$ with respect to the induced coordinates $\left(\xi^{A}\right)=\left(x^{h}, y^{h}, z^{h}\right)$ in each $\pi_{2}^{-1}(U)$. For any point $\mathrm{P}$ of $M$ we denote by $\overline{\mathrm{P}}$ the point of $\bar{M}$ corresponding to $\mathrm{P}$. Then the transformation $\left(S_{\mathrm{P}}\right)^{*}$ induced from $S_{\mathrm{P}}$ (Cf. $\S 1$ ) is a symmetry with center $\overline{\mathrm{P}}$ with respect to $g^{\mathrm{II}}$ (resp. $\nabla^{\mathrm{II}}$ ). On the other hand, $T_{2}(M)$ is homogeneous with respect to $g^{\mathrm{II}}$ (resp. $\nabla^{\mathrm{II}}$ ), because $M$ is so with respect to $g$ (resp. $V$ ). Therefore, taking an arbitrary point $\sigma$ in $T_{2}(M)$, we know that there exists an isometry (resp. an affine transformation) $\tilde{\varphi}$ such that $\tilde{\varphi}(\overline{\mathrm{P}})=\sigma$. Hence, the transformation $\tilde{\varphi} \circ\left(S_{\mathrm{P}}\right) *_{\circ} \tilde{\varphi}^{-1}$ is a symmetry with center $\sigma$, i.e., $T_{2}(M)$ is symmetric with respect to $g^{\text {II }}$ (resp. $\nabla^{\text {II }}$ ). Thus we have

Proposition 8.11. If $M$ is symmetric with respect to a pseudo-Riemannian metric $g$ (resp. an affine connection $V)$, so is $T_{2}(M)$ with respect to $g^{\mathrm{II}}$ (resp. $\nabla^{\mathrm{II}}$ )

\section{§ 9. Geodesics.}

Let $\nabla$ be a torsionless affine connection in $M$. We denote by $\Gamma_{j}{ }_{2}{ }_{\imath}$ the coefficients of $\nabla$ in a coordinate neighborhood $\left(U,\left(x^{h}\right)\right)$ of $M$, where $\Gamma_{j}{ }^{h} \imath_{\imath}=\Gamma_{\imath}{ }^{h}{ }_{\jmath}$. Let $\tilde{C}$ be a curve in $T_{2}(M)$ and suppose that $\widetilde{C}$ is expressed locally by equations

$$
\begin{gathered}
\xi^{A}=\xi^{A}(t), \quad \text { i.e., } \\
x^{h}=x^{h}(t), \quad y^{h}=y^{h}(t), \quad z^{h}=z^{h}(t)
\end{gathered}
$$

with respect to the induced coordinates $\left(\xi^{A}\right)=\left(x^{h}, y^{h}, z^{h}\right)$ in $\pi_{2}^{-1}(U), t$ being a para- 
meter. We now put along $\tilde{C} \cap \pi_{2}^{-1}(U)$

$$
v^{h}=z^{h}+y^{j} y^{i} \Gamma_{j}^{h}
$$

and

$$
\frac{\delta y^{h}}{d t}=\frac{d y^{h}}{d t}+\Gamma_{j}{ }^{h} i \frac{d x^{j}}{d t} y^{2}, \quad \frac{\delta^{2} y^{h}}{d t^{2}}=\frac{d}{d t}\left(\frac{\delta y^{h}}{d t}\right)+\Gamma_{j}{ }^{h} i \frac{d x^{j}}{d t} \frac{\delta y^{2}}{d t}
$$

$$
\frac{\delta v^{h}}{d t}=\frac{d v^{h}}{d t}+\Gamma_{j_{\imath}}{ }_{\imath} \frac{d x^{\jmath}}{d t} v^{2}, \quad \frac{\delta^{2} v^{h}}{d t^{2}}=\frac{d}{d t}\left(\frac{\delta v^{h}}{d t}\right)+\Gamma_{j^{h}}{ }_{\imath} \frac{d x^{\jmath}}{d t} \frac{\delta v^{2}}{d t}
$$

where $x^{h}(t), y^{h}(t)$ and $z^{h}(t)$ are the functions appearing in (9.1). Denoting by $C$ the projection $\pi_{2}(\widetilde{C})$ of $\widetilde{C}$ in $M$, we see that the curve $C$ is expressed as $x^{h}=x^{h}(t)$ in $\left(U,\left(x_{h}\right)\right), x^{h}(t)$ being the functions appearing in (9.1). Then the quantities

$$
y^{h}, v^{h}, \frac{\delta y^{h}}{d t}, \frac{\delta v^{h}}{d t}, \frac{\delta^{2} y^{h}}{d t^{2}}, \frac{\delta^{2} v^{h}}{d t^{2}}
$$

defined above are respectively global vector fields along $C$.

A curve $\tilde{C}$ in $T_{2}(M)$ is a geodesic with respect to $\nabla^{\text {II }}, t$ being an affine parameter, if and only if its local expression (9.1) satisfies the differential equations

$$
\begin{aligned}
& \frac{d^{2} \xi^{A}}{d t^{2}}+\tilde{\Gamma}_{C^{A}}{ }^{A} \frac{d \xi^{C}}{d t} \frac{d \xi^{B}}{d t}=0, \quad \text { i.e., } \\
& \frac{d^{2} x^{h}}{d t^{2}}+\tilde{\Gamma}_{C^{h}}{ }_{B} \frac{d \xi^{C}}{d t} \cdot \frac{d \xi^{B}}{d t}=0
\end{aligned}
$$

(9. 4)

$$
\begin{aligned}
& \frac{d^{2} y^{h}}{d t^{2}}+\tilde{\Gamma}_{C^{\bar{h}}}{ }_{B} \frac{d \xi^{C}}{d t} \frac{d \xi^{B}}{d t}=0 \\
& \frac{d^{2} z^{h}}{d t^{2}}+\tilde{\Gamma}_{C^{\prime}} \overline{\bar{h}}_{B} \frac{d \xi^{C}}{d t} \frac{d \xi^{B}}{d t}=0
\end{aligned}
$$

where $\Gamma_{C}{ }_{B}, \Gamma_{C} \bar{h}_{B}$ and $\Gamma_{C} \overline{\bar{h}}_{B}$ are the coefficients of $\nabla^{\text {II }}$ given by (7.1), (7.2) and (7. 3). The equations (9.4) are equivalent to the equations

$$
\begin{aligned}
& \frac{d^{2} x^{h}}{d t^{2}}+\Gamma_{j}{ }_{i} \frac{d x^{\jmath}}{d t} \frac{d x^{2}}{d t}=0, \\
& \frac{d^{2} y^{h}}{d t^{2}}+\left(y^{s} \partial_{s} \Gamma_{j}{ }_{j}^{h}\right) \frac{d x^{\jmath}}{d t} \frac{d x^{\imath}}{d t}+2 \Gamma_{j}^{h}{ }_{i} \frac{d x^{\jmath}}{d t} \frac{d y^{\imath}}{d t}=0,
\end{aligned}
$$


(9. 7)

$$
\frac{d^{2} z^{h}}{d t^{2}}+\left(z^{s} \partial_{s} \Gamma_{j}{ }^{h}{ }_{i}+y^{t} y^{s} \partial_{t} \partial_{s} \Gamma_{j}{ }^{h}{ }_{\imath}\right) \frac{d x^{j}}{d t} \frac{d x^{2}}{d t}
$$

$$
+4\left(y^{s} \partial_{s} \Gamma_{j}{ }_{i}\right) \frac{d x^{\jmath}}{d t} \frac{d y^{\imath}}{d t}+2 \Gamma_{j}{ }_{i} \frac{d y^{\jmath}}{d t} \frac{d y^{2}}{d t}+2 \Gamma_{j}{ }_{i} \frac{d x^{\jmath}}{d t} \cdot \frac{d z^{\imath}}{d t}=0 .
$$

Making use of (9.2) and (9.3), we see that the system of differential equations (9. 5), (9.6) and (9.7) is equivalent to the the system of differential equations

$$
\begin{gathered}
\frac{d^{2} x^{h}}{d t^{2}}+\Gamma_{j}^{h} \frac{d x^{\jmath}}{d t} \frac{d x^{2}}{d t}=0 \\
\frac{\delta^{2} y^{h}}{d t^{2}}+R_{k j i}{ }^{h} y^{k} \frac{d x^{\jmath}}{d t} \frac{d x^{\imath}}{d t}=0 \\
\frac{\delta^{2} v^{h}}{d t^{2}}+R_{k j i}{ }^{h} v^{k} \frac{d x^{j}}{d t} \frac{d x^{2}}{d t}+4 R_{k j i}{ }^{h} y^{k} \frac{d x^{\jmath}}{d t} \frac{\delta y^{2}}{d t} \\
+\left(\nabla_{t} R_{s j i}{ }^{h}-\nabla_{j} R_{i t s}{ }^{h}\right) y^{t} y^{s} \frac{d x^{j}}{d s} \frac{d x^{\imath}}{d s}=0,
\end{gathered}
$$

where $R_{k j i}{ }^{h}$ denote the components of the curvature tensor of $\nabla$. That is to say, the system of differential equations (9.8), (9.9) and (9.10) determines in $T_{2}(M)$ geodesics with respect to the affine connection $\nabla^{\mathrm{II}}$. Thus we have

Proposition 9. 1. Let $\tilde{C}$ be a geodesic in $T_{2}(M)$ with respect to $\nabla^{\mathrm{II}}$, where $\nabla$ is a torsionless affine connection in $M$, and suppose that $\tilde{C}$ has the local expression (9. 1). Then the projection $C=\pi_{2}(\tilde{C})$ is a geodesic in $M$ with respect to $\nabla$. The vector field $y^{h}(t)$ defined along $C$ is a Jacobi field with respect to $\nabla$. The vector field $v^{h}(t)$ defined by (9.2) along $C$ satisfies the differential equation (9.10). The affine parameter of $\tilde{C}$ induces naturally an affine parameter along $C$.

Conversely, if there exists in $M$ a geodesic with respect to $\nabla, C$ having the local expression $x^{h}=x^{h}(t)$ with affine parameter $t$, if there is given a Jacobi vector field $y^{h}(t)$ along $C$, and, if there is given a vector field $v^{h}(t)$ satisfying along $C$ the differential equation (9.10), then the curve $\tilde{C}$ defined in $T_{2}(M)$ by the local expression $x^{h}=x^{h}(t), y^{h}=y^{h}(t), z^{h}=v^{h}(t)-y^{j}(t) y^{2}(t) \Gamma_{j}{ }^{h} i\left(x^{s}(t)\right)$ is a geodesic in $T_{2}(M)$ with respect to $\nabla^{\mathrm{II}}$.

Taking account of (9.8), (9.9) and (9.10) we see easily that, if there is given in $M$ a geodesic $C$ with respect to a torsionless affine connection $\nabla, C$ having the local expression $x^{h}=x^{h}(t)$, and a Jacobi field $v^{h}(t)$ along $C$, then the curve $\widetilde{C}$ defined in $T_{2}(M)$ by the local expression $x^{h}=x^{h}(t), y^{h}=0, z^{h}=v^{h}(t)$ is a geodesic with respect to $\nabla^{\mathrm{II}}$, 
We say that $M$ is complete with respect to an affine connection (resp. a pseudoRiemannian metric $g$ ) if along any geodesic any affine parameter takes an arbitrarily given real value. Then, taking account of (9.8), (9.9) and (9. 10), we have

Proposition 9.2. If $M$ is complete with respect to a torsionless affine connection $\nabla$ (resp. a pseudo-Riemannian metric $g$ ), so is $T_{2}(M)$ with respect to $\nabla^{\mathrm{II}}$ (resp. $\left.g^{\mathrm{II}}\right)$.

According to [15], we have from (9.8), (9.9) and (9.10)

Proposition 9. 3. Let $\tilde{C}$ be a geodesic in $T_{2}(M)$ with respect to $\nabla^{\mathrm{II}}, \nabla$ being a torsionless affine connection in $M$. Then the projection $\pi_{12}(\widetilde{C})$ of $\tilde{C}$ in the tangent bundle $T_{1}(M)$ is also a geodesic with respect to $\nabla^{C}$, where $\nabla^{C}$ is the complete lift of the affine connection $\nabla$ in the sense of [15].

\section{BIBLIOGRAPHY}

[1] Dombrowski, P., On the geometry of the tangent bundle. J. Reine und Angew. Math. 210 (1962), 73-88.

[2] Ledger, A. J., AND K. YAno, The tangent bundle of a locally symmetric space. J. London Math. Soc. 40 (1965), 487-492.

[3] Ledger, A. J., And K. Yano, Almost complex structures on tensor bundles. Journal of Differential Geometry. 1 (1967), 355-368.

[4] Morimoto, A., Note on prolongations of affine connections to tangent bundles. To appear.

[5] Morimoto, A., Prolongations of $G$-structures to tangent bundles. To appear.

[6] SASAKI, S., On the differential geometry of tangent bundles of Riemannian manifolds. Tôhoku Math. J. 10 (1958), 338-354.

[7] Satô, I., Almost analytıc vector fields in almost complex manifolds. Tôhoku Math. J. 17 (1965), 185-199.

[8] Tachibana, S., And M. OKumura, On the almost complex structure of tangent bundles of Riemannıan spaces. Tôhoku Math. J. 14 (1962), 156-161.

[9] Tanno, S., An almost complex structure of the tangent bundle of an almost contact manifold. Tôhoku Math. J. 17 (1965), 7-15.

[10] Tondeur, P., Structure presque kählérienne sur la fibré des vecteurs covarıants d'une variéte riemannienne. C. R. Acad. Sc1. Paris 254 (1962), 407-408.

[11] Yano, K., Tensor fields and connections on cross-sections in the tangent bundle of a differentiable manifold. Proc. Royal Soc. Edinburgh, Ser. A, 67 (1967), $277-288$.

[12] YANo, K., Tensor fields and connections on cross-section in the cotangent bundle. Tôhoku Math. J. 19 (1967), 32-48.

[13] Yano, K., and E. T. Davies, On the tangent bundles of Finsler and Riemannian manifold. Rend. Cir. Math. Palermo, Series II, 12 (1963), 1-18.

[14] Yano, K., AND S. Ishinara, Almost complex structures induced in tangent bundles. Kōdai Math. Sem. Rep. 19 (1967), 1-27.

[15] Yano, K., and S. Kobayashi, Prolongations of tensor fields and connections to 
tangent bundle, I, II. J. Math. Soc. Japan 186 (1966), 194-210, 236-246.

[16] YANo, K., AND A. J. Ledger, Linear connections on tangent bundles. J. London Math. Soc. 39 (1964), 495-500.

[17] Yano, K., and E. M. Patterson, Vertical and complete lifts from a manifold to its cotangent bundle. J. Math. Soc. Japan 19 (1967), 91-113.

[18] Yano, K., and E. M. Patterson, Horizontal lifts from a manifold to its tangent bundle. J. Math. Soc. Japan 19 (1967), 185-198.

Department of Mathematics,

Tokyo Institute of Technology. 